\title{
Schwann cell-derived periostin promotes autoimmune peripheral polyneuropathy via macrophage recruitment
}

\author{
Denise E. Allard, ${ }^{1}$ Yan Wang, ${ }^{2}$ Jian Joel Li, ${ }^{3}$ Bridget Conley, ${ }^{2}$ Erin W. Xu, ${ }^{2,4}$ David Sailer, ${ }^{2}$ Caellaigh Kimpston, ${ }^{2}$ Rebecca Notini, ${ }^{2}$ \\ Collin-Jamal Smith, ${ }^{1}$ Emel Koseoglu, ${ }^{5,6}$ Joshua Starmer, ${ }^{7}$ Xiaopei L. Zeng, ${ }^{2}$ James F. Howard Jr., ${ }^{8}$ Ahmet Hoke, ${ }^{5}$ Steven S. Scherer, ${ }^{3}$ \\ and Maureen A. Su $\mathbf{u}^{1,2,4,9}$ \\ 'Department of Microbiology and Immunology and ${ }^{2}$ Department of Pediatrics, University of North Carolina at Chapel Hill (UNC-CH), Chapel Hill, North Carolina, USA. ${ }^{3}$ Department of Neurology, University of \\ Pennsylvania, Philadelphia, Pennsylvania, USA. ํㅡepartment of Microbiology, Immunology, and Molecular Genetics, UCLA, Los Angeles, California, USA. ${ }^{5}$ Department of Neurology, Johns Hopkins University, \\ Baltimore, Maryland, USA. ${ }^{6}$ Neurology Department, School of Medicine, Erciyes University, Kayseri, Turkey. ${ }^{7}$ Department of Genetics and ${ }^{8}$ Department of Neurology, UNC-CH, Chapel Hill, North Carolina, USA. \\ 9Department of Pediatrics, UCLA, Los Angeles, California, USA.
}

\begin{abstract}
Chronic inflammatory demyelinating polyneuropathy (CIDP) and Guillain-Barre syndrome (GBS) are inflammatory neuropathies that affect humans and are characterized by peripheral nerve myelin destruction and macrophage-containing immune infiltrates. In contrast to the traditional view that the peripheral nerve is simply the target of autoimmunity, we report here that peripheral nerve Schwann cells exacerbate the autoimmune process through extracellular matrix (ECM) protein induction. In a spontaneous autoimmune peripheral polyneuropathy (SAPP) mouse model of inflammatory neuropathy and CIDP nerve biopsies, the ECM protein periostin (POSTN) was upregulated in affected sciatic nerves and was primarily expressed by Schwann cells. Postn deficiency delayed the onset and reduced the extent of neuropathy, as well as decreased the number of macrophages infiltrating the sciatic nerve. In an in vitro assay, POSTN promoted macrophage chemotaxis in an integrin-AM (ITCAM) and ITCAV-dependent manner. The PNS-infiltrating macrophages in SAPP-affected nerves were pathogenic, since depletion of macrophages protected against the development of neuropathy. Our findings show that Schwann cells promote macrophage infiltration by upregulating Postn and suggest that POSTN is a novel target for the treatment of macrophageassociated inflammatory neuropathies.
\end{abstract}

\section{Introduction}

Chronic inflammatory demyelinating polyneuropathy (CIDP) and acute inflammatory demyelinating polyneuropathy (AIDP, a form of Guillain-Barre syndrome [GBS]) are characterized by sensory impairment and disabling weakness $(1,2)$ and are the result of autoimmune demyelination of the PNS. Current therapies include plasmapheresis and intravenous Ig (IVIg) for CIDP and AIDP as well as corticosteroids for CIDP, all of which have nonspecific mechanisms of action and broadly suppress the immune system $(3,4)$. Patients with CIDP often require long-term treatment in order to maintain therapeutic effect (3), and even with these treatments, clinical remission is not achieved in approximately $30 \%$ of patients (5). Thus, the development of novel, mechanism-based therapies is desirable and would be greatly aided by the delineation of pathogenic mechanisms.

Through the use of mouse models of inflammatory neuropathies and patients' biopsy samples, the immune mechanisms that contribute to the development of autoimmune peripheral neuropathy have been partially illuminated. The prominence of $\mathrm{T}$ cells and $\mathrm{F} 4 / 80^{+}$macrophages in affected nerves of spontaneous autoimmune peripheral polyneuropathy (SAPP) mice $(6,7)$, experimental allergic neuritis (EAN) mice $(8,9)$, and human patients $(10,11)$ suggests that these cells are particularly important. Fur-

Authorship note: DEA and YW are co-first authors.

Conflict of interest: The authors have declared that no conflict of interest exists.

Submitted: December 18, 2017; Accepted: July 31, 2018

Reference information: / Clin Invest. 2018;128(10):4727-4741.

https://doi.org/10.1172/JCI99308. ther, in SAPP and EAN, CD4 ${ }^{+}$T cells isolated from neuropathic mice are sufficient to transfer disease to $\mathrm{T}$ and $\mathrm{B}$ cell-deficient (SCID) recipients, underscoring an important role for $\mathrm{CD}^{+} \mathrm{T}$ cells among lymphocytes $(7,12,13)$. Both Th1 and Th17 have been implicated in the pathogenesis of CIDP and GBS, since the frequency of Th1 and Th17 cells is elevated in the blood and cerebrospinal fluid (CSF) of patients $(14,15)$, but the relative importance of Th1 and Th17 is still controversial. In models of SAPP, Th1 T cells predominate $(7,12)$, but in EAN, an important role for Th17 has also been suggested (16). Macrophages may also contribute to autoimmune destruction of the PNS, since macrophages in EAN exhibit an inflammatory phenotype, which includes the secretion of proinflammatory factors such as ROS and the cytokines IL-12, TNF- $\alpha$, and IL-6 (17). The signals that recruit CD4 $4^{+} \mathrm{T}$ cells and macrophages into the PNS in inflammatory neuropathies, however, are currently unclear.

The extracellular matrix (ECM) is a prominent feature of the PNS and has been implicated in numerous developmental processes, including Schwann cell migration, radial sorting of axons, and myelination $(18,19)$. In light of recent reports that found a role for ECM proteins in inflammation (20-24), we investigated their possible role in the development of SAPP (7). We found increased expression of the ECM protein periostin (POSTN) by Schwann cells in affected nerves of SAPP mice and patients with CIDP. Moreover, Postn expression aggravates neuropathy, since Postn-deficient animals have delayed disease onset and improved nerve function by both electrophysiological and pathological analysis. Additionally, Postn-deficient sciatic nerves show reduced 
infiltration of both $\mathrm{T}$ cells and macrophages. Macrophages, but not $\mathrm{T}$ cells, migrated across a Transwell membrane in response to POSTN, suggesting that POSTN primarily induces macrophage chemotaxis. Finally, macrophages recruited to affected sciatic nerves are pathogenic, as macrophage-depleted animals showed delayed disease onset and improved nerve function. Overall, our results demonstrate that Schwann cell-derived POSTN promotes neuropathy pathogenesis by directly recruiting pathogenic macrophages to the PNS.

\section{Results}

Postn expression is induced during SAPP. To investigate a possible role for ECM proteins in the development of inflammatory neuropathy, we used a strain of NOD mice that develops SAPP, owing to a dominant, loss-of-function $\mathrm{G} 228 \mathrm{~W}$ mutation in the autoimmune regulator gene (NOD.Aire ${ }^{G W /+}$ mice) (7). PNS autoimmunity in NOD.Aire ${ }^{G W /+}$ mice shares key features with human CIDP, including infiltration of peripheral nerves by $\mathrm{CD} 4^{+} \mathrm{T}$ cells and $\mathrm{F} 4 / 80^{+}$macrophages (25-27), IFN- $\gamma$ production by $\mathrm{CD}^{+}{ }^{+} \mathrm{T}$ cells $(28,29)$, and peripheral nerve demyelination $(7,30)$. We screened mRNA levels of 6 ECM genes with known roles in PNS development: laminin a2 (Lama2) (31), laminin a4 (Lama4) (31), laminin a5 (Lama5) (32), laminin b1 (Lamb1) (31), thrombospondin 2 (Thbs2) (33), and Postn $(34,35)$. We used quantitative reverse transcription PCR (qRT-PCR) to compare the relative expression levels of these genes between sciatic nerves from age-matched WT (NOD. WT) mice and those of affected NOD.Aire ${ }^{G W /+}$ mice. Sciatic nerves from NOD.Aire ${ }^{G W /+}$ mice were harvested when severe neuropathy (clinical score of 3 out of a maximum of 4 ) was observed. While the levels of Lama2, Lama4, Lama5, and Lamb1 mRNA showed no change (Figure 1, A-D), the levels of Thbs 2 and Postn were significantly increased in neuropathic NOD.Aire ${ }^{G W /+}$ mice compared with levels in NOD.WT mice (Figure 1, E and F). Increased Thbs2 expression with PNS inflammation was not surprising, given previous reports that Thbs 2 expression increases after nerve injury and that Thbs 2 may play a role in axonal regeneration (36). However, increased Postn expression was unexpected, since it has not been previously linked to peripheral nerve injury or inflammation.

A member of the fasciclin family, Postn is widely expressed during embryonic development $(34,37-40)$. POSTN promotes cell motility through interaction with integrins $(41,42)$ and has been linked to several inflammatory diseases (20-22, 43), making it an intriguing target to investigate. Increased Postn expression was associated with neuropathy, since 8-week-old preneuropathic mice expressed similar levels of Postn mRNA compared with levels in NOD.WT mice (Supplemental Figure 1; supplemental material available online with this article; https://doi.org/10.1172/ JCI99308DS1). To examine POSTN expression in SAPP, we used Western blotting and immunostaining to characterize POSTN protein expression in the PNS of neuropathic mice. Western blot analysis showed significantly increased POSTN protein in whole sciatic nerves from neuropathic NOD.Aire ${ }^{G W /+}$ mice compared with nerves from NOD.WT mice (Figure $1, \mathrm{G}$ and $\mathrm{H}$ ). We verified that this $75-\mathrm{kDa}$ band corresponded to POSTN, since it was absent in Postn-deficient mice (Supplemental Figure 2). Immunostaining showed that POSTN immunoreactivity was mostly restricted to the perineurium of NOD.WT nerves (Figure 1I, top left), whereas
POSTN immunoreactivity was also diffusely positive in the endoneurium of affected NOD.Aire ${ }^{G W /+}$ nerves (Figure 1I, top right). Thus, Postn mRNA and protein expression is increased in neuropathic NOD.Aire $\mathrm{GW/+}^{\mathrm{W}}$ nerves.

To determine whether increased Postn expression is specific to NOD.Aire ${ }^{G W /+}$ mice, we immunostained sciatic nerves from 2 additional models of inflammatory neuropathies: (a) autoimmune peripheral neuropathy induced in immunodeficient SCID mice upon transfer of splenocytes from neuropathic NOD.Aire ${ }^{G W /+}$ mice (7) and (b) transfer of $\mathrm{CD}^{+} \mathrm{T}$ cells from a myelin protein zerospecific (Mpz- or PO-specific) T cell receptor-Tg mouse line (NOD. POT TCR-Tg) (44). Reconstituted mice were sacrificed when severe neuropathy (clinical score of 3) was observed. We observed by immunohistochemistry that POSTN expression in sciatic nerves was increased in both of these models (Figure 1I, bottom 2 images), suggesting that induction of Postn is not unique to NOD. Aire $^{\mathrm{GW} / \mathrm{+}}$ mice, but is a more generalizable feature of inflammatory peripheral neuropathies in mice. Interestingly, we observed no change in the expression of Postn 3 days after axotomy (Supplemental Figure 3), suggesting that Postn upregulation in the PNS may be specific to inflammatory neuropathies. Finally, to determine whether POSTN is upregulated in human CIDP, we immunostained nerve biopsies from 5 patients with CIDP and 5 patients with axonal (noninflammatory) neuropathy. We detected POSTN expression in 2 of the 5 CIDP patients' samples, but not in the samples from the patients with axonal neuropathy (Figure 1J). Therefore, POSTN upregulation is a feature of inflammatory neuropathy in both mice and humans.

Schwann cells express Postn during SAPP. To identify the cells expressing Postn, we generated NOD Postn reporter mice, which harbor a Postn allele into which a lacZ cassette is targeted (45). We performed histochemistry for $\beta$-gal using X-gal as a substrate (46) on nerves from neuropathic NOD.Aire ${ }^{G W /+}$ and age-matched NOD.WT mice that were heterozygous for this Postn allele (Postn $\left.n^{\text {lacz/+ }}\right)$. In NOD.WT Postn ${ }^{\text {lacz/+ }}$ mice, X-gal crystals were restricted to the perineurium of sciatic nerves (Figure 2A, top left), but in severely neuropathic (clinical score of 3) NOD.Aire ${ }^{G W /+}$ Postn ${ }^{\text {lacZ/+ }}$ mice, X-gal crystals were also prominent in the endoneurium (Figure 2, A and B). In parallel, we used adoptive transfer (AT) of splenocytes from neuropathic NOD.Aire ${ }^{G / /+}$ mice to induce neuropathy in $\mathrm{T}$ and $\mathrm{B}$ celldeficient NOD.SCID Post ${ }^{\text {lacz/+ }}$ recipients; in reconstituted mice, non-T and non-B cell lineages derived from NOD.SCID hosts harbor the Postn reporter allele. Sciatic nerves from NOD.SCID Postn ${ }^{\text {lacz/+ }}$ recipient mice were examined when severe neuropathy (clinical score of 3) was observed and were compared with unreconstituted NOD.SCID Postn ${ }^{\text {lacz/+ }}$ control mice. In the AT model, we observed $\beta$-galactivityin a pattern that was similarto that of the spontaneous NOD.Aire ${ }^{G W /+}$ model. Namely, in unreconstituted, non-neuropathic control mice, we found that $\beta$-gal activity was absent in the endoneurium and was only seen in the perineurium. On the other hand, in reconstituted, neuropathic recipient mice, we found that $\beta$-gal activity was present in the endoneurium (Figure 2, A and B, bottom), confirming that endoneurial Postn expression was associated with neuropathy development and demonstrating that nonlymphocytes expressed Postn.

Light microscopic analysis of semithin sections of epoxyembedded nerves from both neuropathic NOD.Aire ${ }^{G W /+}$ and NOD. 
A

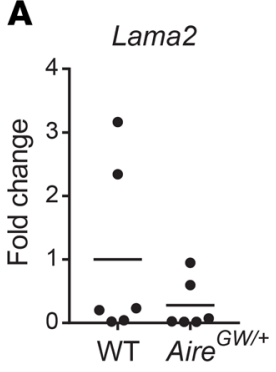

B

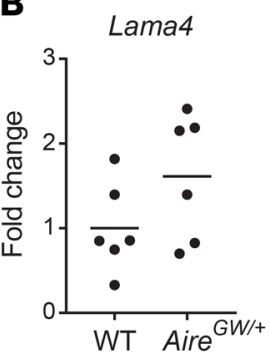

C

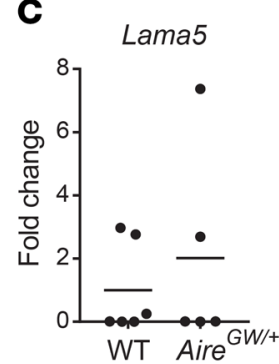

D

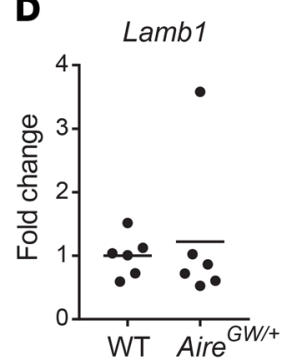

E

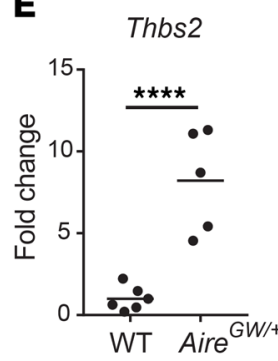

F

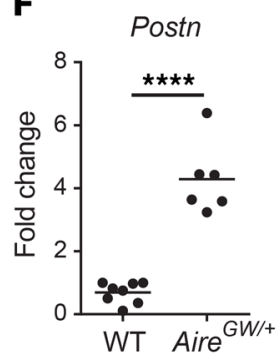

G

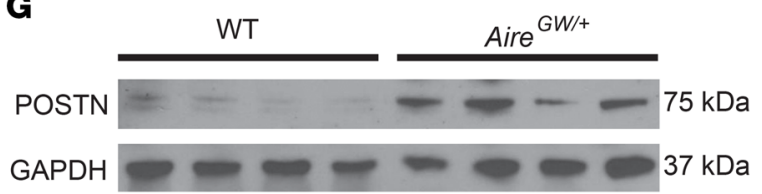

J

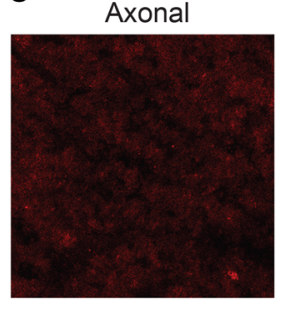

CIDP

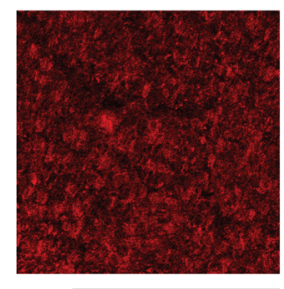

H

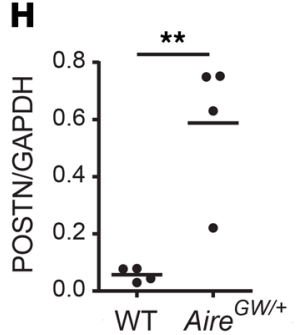

I

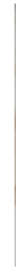
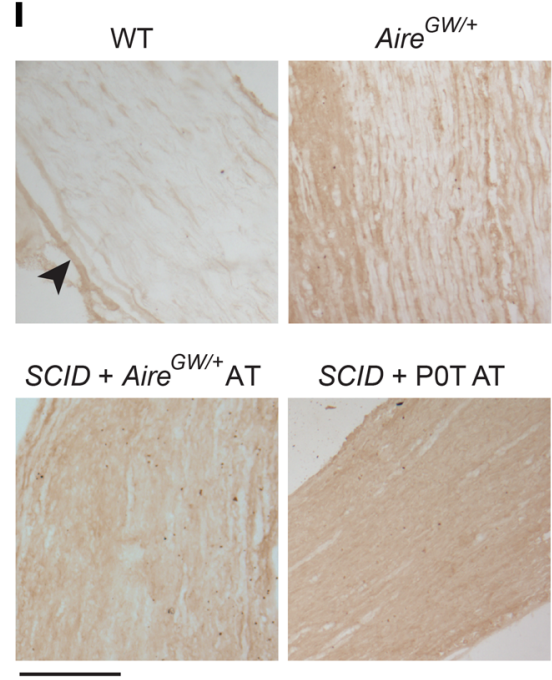

$S C I D+$ POT AT

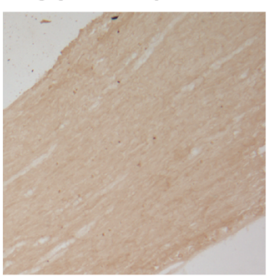

Figure 1. Increased Postn expression in SAPP. (A-F) RNA was isolated from the sciatic nerves of NOD.WT (WT) and NOD.Aire $\mathrm{CW/+}$ neuropathic mice. Lama2 (A), Lama4 (B), Lama5 (C), Lamb1 (D), Thbs2 (E), and Postn (F) expression relative to cyclophilin was measured by qRT-PCR. Values are expressed as the fold change compared with WT. (C) POSTN expression was measured by Western blotting of sciatic nerve lysates from NOD.WT (WT) and NOD.Aire CW/ $^{-}$ $\left(\right.$ Aire $\left.{ }^{C W /+}\right)$ neuropathic mice. GAPDH was used as a loading control. Each lane represents an individual mouse. (H) Densitometric analysis of the Western blot in G. (I) POSTN immunohistochemical staining of sciatic nerves from WT mice, neuropathic NOD. Aire ${ }^{C W /+}$ mice, neuropathic SCID recipients of NOD. Aire ${ }^{\mathrm{CW} / \mathrm{t}}$ splenocytes, and neuropathic SCID recipients of POT splenocytes. Note that POSTN immunoreactivity was mostly found in the perineurium (arrowheads) of NOD.WT (WT) nerves, whereas the endoneurium was diffusively positive in nerves from NOD.Aire ${ }^{C W /+}$ mice, SCID recipients of NOD.Aire ${ }^{C W /+}$ splenocyte AT, and SCID recipients of NOD.POT splenocyte AT. Scale bar: $180 \mu \mathrm{m}$. (J) Immunofluorescence staining of biopsy samples from patients with axonal neuropathy or CIDP. Increased endoneurial POSTN immunoreactivity was observed in the CIDP sample. Scale bar: $200 \mu \mathrm{m}$. (A-F and H) Each dot represents an individual animal. ${ }^{* *} P<0.005$ and ${ }^{* * *} P<0.0001$, by 2 -tailed, unpaired $t$ test. Individuals values and means are shown.

SCID Postn ${ }^{\text {lacz/+ }}$ AT recipient mice revealed diffuse endoneurial $\beta$-gal activity as well as areas of intense $\beta$-gal activity (Figure $2 \mathrm{~B}$ ). We next used electron microscopy (EM) to characterize the cell types expressing Postn in the endoneurium of neuropathic nerves. EM revealed that X-gal crystals were in neuropathic NOD.Aire ${ }^{G W /+}$ Schwann cells, which was inferred by their physical relationships with axons (Figure 2, C and D, top panels). We observed a similar pattern in nerves from reconstituted, neuropathic NOD.SCID Post $n^{\text {lac } / /+}$ recipient mice (Figure 2, C and D, bottom, and Figure 2E). We observed X-gal crystals in promyelinating Schwann cells that ensheathed large axons (Figure 2C), nonmyelinating Schwann cells associated with many small, unmyelinated axons (Figure 2D), and normal-appearing, myelinating Schwann cells (Figure 2E). We also observed X-gal crystals in endoneurial cells with features distinct from those of Schwann cells (Supplemental Figure 4). To rule out the possibility that X-gal crystals were a nonspecific staining artifact independent of the lac $Z$ transgene, we performed histochemistry for $\beta$-gal on NOD.SCID mice lacking the Postn ${ }^{\text {lacZ }}$ reporter. Nerve sections from NOD.SCID Postn ${ }^{+/+}$recipient mice did not exhibit X-gal crystals, even in regions with reduced numbers of myelinated axons (Supplemental Figure 5), demonstrating that $\mathrm{X}$-gal crystals are specific to the lac $Z$ transgene. Together, these findings indicate that X-gal crystals seen in Schwann cells and non-Schwann endoneurial cells of neuropathic NOD. Aire ${ }^{G W /+}$ mice reflect Postn reporter expression.

To confirm that Schwann cells are a source of increased Postn expression in neuropathic nerves, we measured relative Postn mRNA expression by qRT-PCR in flow-sorted live CD45 $\mathrm{CD}^{-} \mathrm{p} 75^{+}\left(\mathrm{p} 75^{+}\right)$Schwann cells (gating strategy in Supplemental Figure 6). In NOD.WT mice, in which neuropathy is absent, this cell population consists of nonmyelinating Schwann cells (47). In neuropathic NOD.Aire $\mathrm{GW/+}^{\mathrm{T}}$ mice, on the other hand, this cell population probably also contains denervated $(48,49)$ and promyelinating (50) Schwann cells in addition to the normal nonmyelinating Schwann cells. In line with our EM results, $\mathrm{p} 75^{+}$ Schwann cells from neuropathic NOD.Aire ${ }^{\mathrm{GW} /+}$ mice expressed significantly more Postn mRNA than did $\mathrm{p} 75^{+}$Schwann cells isolated from NOD.WT animals (Figure 2F). Since X-gal crystals were also observed in non-Schwann cells (Supplemental Figure 4), we also measured Postn expression by qRT-PCR in $\mathrm{CD}^{+} \mathrm{T}$ 
A

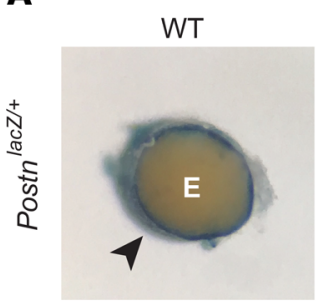

No AT

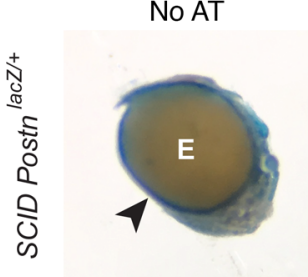

Aire ${ }^{\text {GW/+ }}$

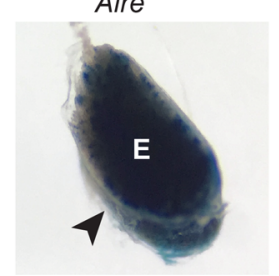

AT

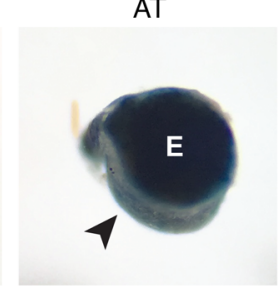

C
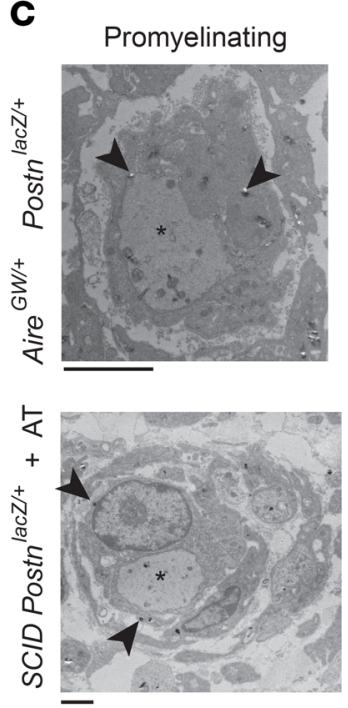

$\mathbf{F}$

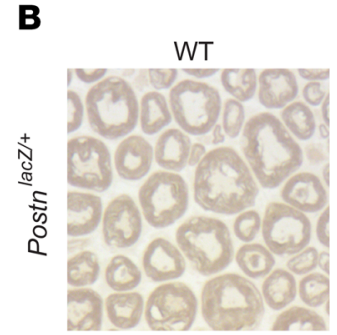

No AT

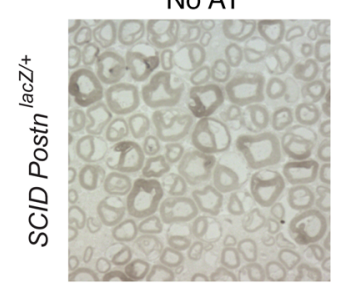

Aire $^{\text {GW/+ }}$

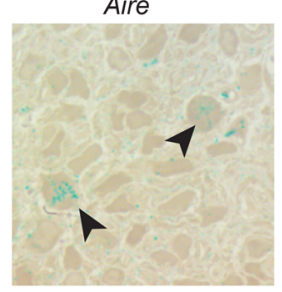

AT

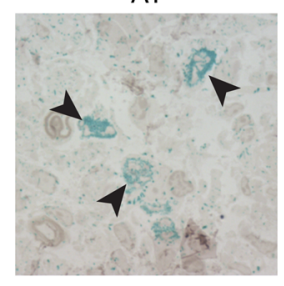

G

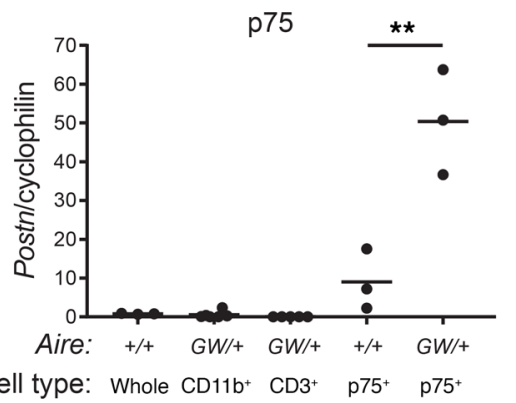

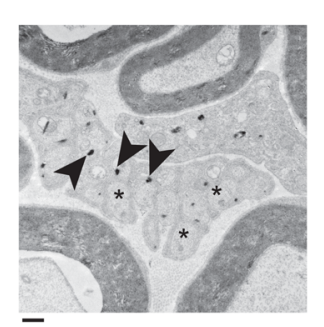

E

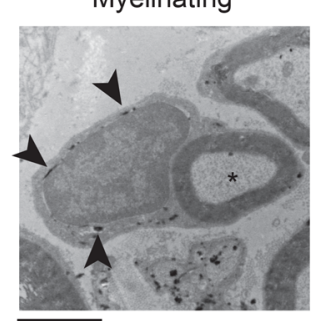

H
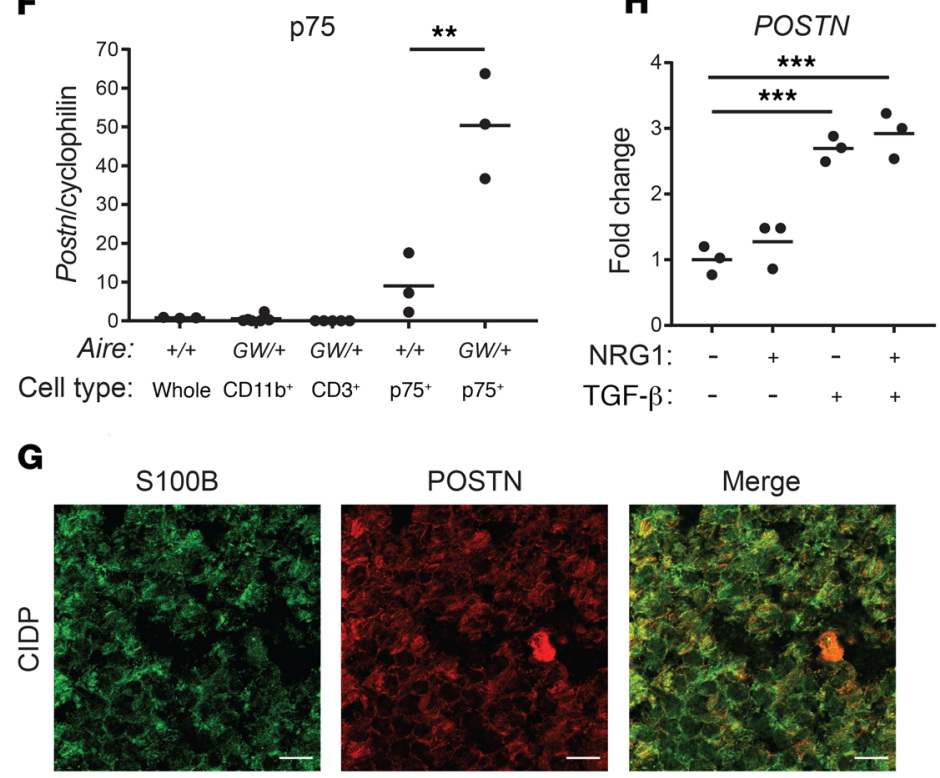

Figure 2. Postn is expressed by Schwann cells. Histochemistry for $\beta$-gal using X-gal was performed on sciatic nerves from NOD.WT Postn ${ }^{\text {lacz/+ }}$ mice (WT, $n=4)$ and NOD. Aire ${ }^{C W /+}$ Postn ${ }^{\prime a c z /+}$ mice $(n=3)$ as well as from NOD.SCID Postn ${ }^{1 a c Z /+}\left(\right.$ Posth $\left.{ }^{l a c Z /+}\right)$ reporter mice without $(n=4)$ or with $(n=8)$ NOD.Aire ${ }^{C W /+}$

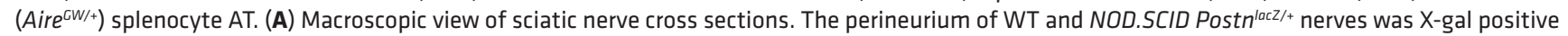
(arrowheads); the endoneurium (E) was also X-gal positive in NOD. Aire ${ }^{C W /+}$ Postn $n^{\text {lacz/+ }}$ and NOD.SCID Postn ${ }^{l a c z /+}$ mice that underwent AT. Original magnification, $\times 4.5$. (B) Semithin sections were stained with paraphenylenediamine. X-gal crystals are blue, and some large clusters are indicated with arrowheads in a region with reduced myelinated axon density. Original magnification, $\times 100$. (C-E) EM images. Arrowheads indicate X-gal crystals, which are electron dense; asterisks indicate axons. X-gal crystals were found in promyelinating (C), nonmyelinating (D), and myelinating (E) Schwann cells. Scale bars: $2 \mu \mathrm{m}$ (C and E) and $500 \mathrm{~nm}$ (D). (F) Postn expression relative to cyclophilin, measured by qRT-PCR. RNA was isolated from whole sciatic nerves from NOD.WT mice, CD11 b+ or $\mathrm{CD}^{+}$cells enriched from NOD. Aire ${ }^{\mathrm{CW} /+}$ neuropathic mice, or $\mathrm{p} 75^{+}$Schwann cells purified from sciatic nerves from WT and affected NOD. Aire ${ }^{\mathrm{CW} /+}$ neuropathic mice. Each dot in $\mathbf{F}$ represents an individual mouse. (C) Immunofluorescence staining of S100B (green) and POSTN (red) in CIDP patients' biopsy samples. Scale bar: $20 \mu \mathrm{m}$. (H) HSCs were cultured with NRG1, TGF- $\beta$, or both NRG1 and TGF- $\beta$. POSTN expression was measured by qRT-PCR. Each dot in $\mathbf{H}$ represents an individual well. ${ }^{* *} P<0.005$ and ${ }^{* *} P<0.0005$, by 1-way ANOVA with multiple comparisons test.

cells and $\mathrm{CD} 11 \mathrm{~b}^{+}$macrophages isolated from the sciatic nerve of neuropathic NOD.Aire ${ }^{G W /+}$ mice. Neither $\mathrm{CD}^{+} \mathrm{T}$ cells nor $\mathrm{CD}_{11} \mathrm{~b}^{+}$ macrophages expressed Postn (Figure 2F). Thus, we conclude that Schwann cells induce Postn expression in mouse models of inflammatory neuropathies and are the main source of POSTN in the sciatic nerve during neuropathy, whereas $\mathrm{CD}^{+} \mathrm{T}$ cells and $\mathrm{CD}_{11 \mathrm{~b}}{ }^{+}$macrophages do not express Postn.
While our data indicate that murine Schwann cells produce POSTN during neuropathy, the cellular source of POSTN in patients with CIDP is still unclear. To determine whether human Schwann cells (HSCs) are the source of POSTN in patients with CIDP, we immunofluorescently labeled patients' CIDP biopsy samples with antibodies against POSTN and the Schwann cell marker S10OB (Figure 2G). We observed prominent overlap 
A

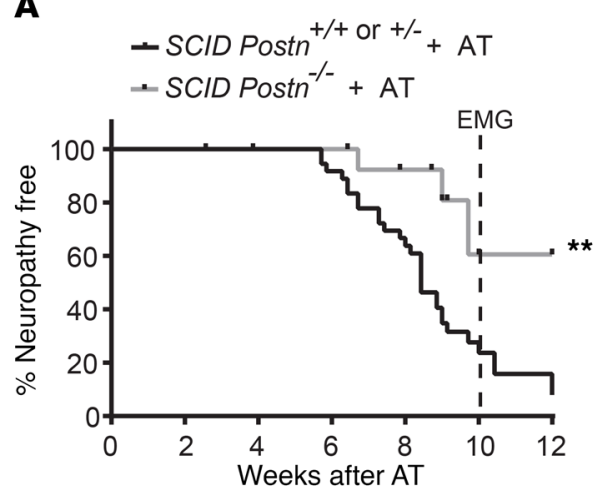

$\mathbf{F}$

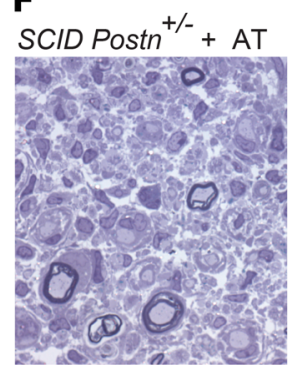

G

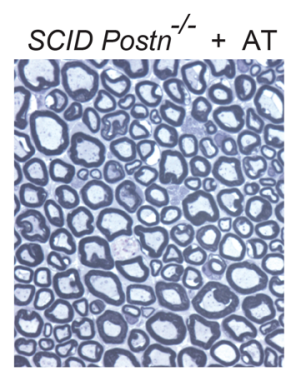

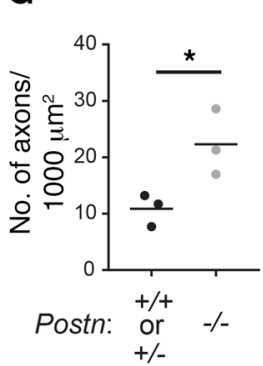

B

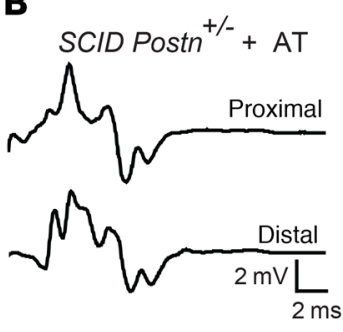

C

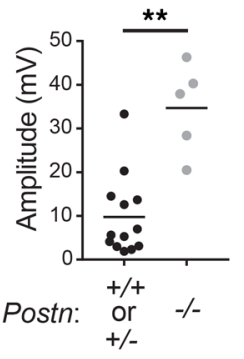

D

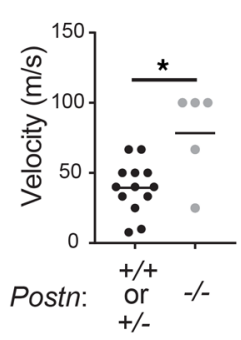

SCID Postn ${ }^{-1-}+$ AT
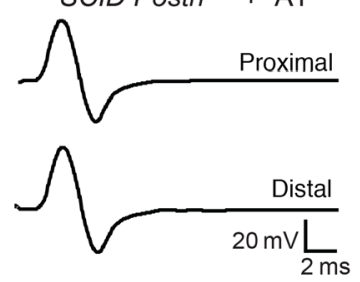

E

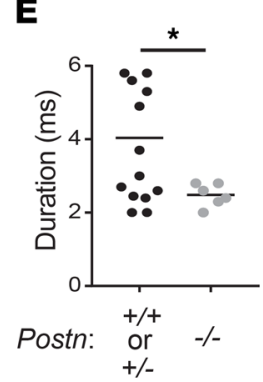

H

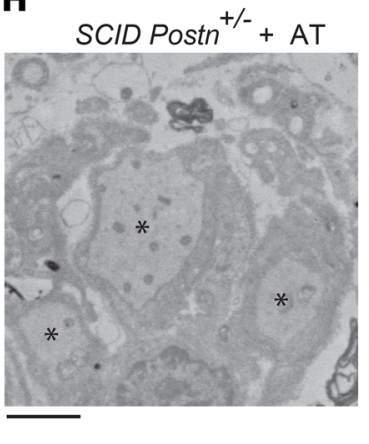

SCID Postn ${ }^{-/-}+$AT

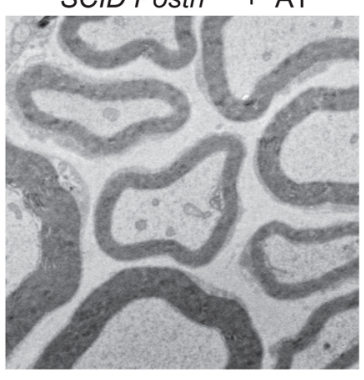

I

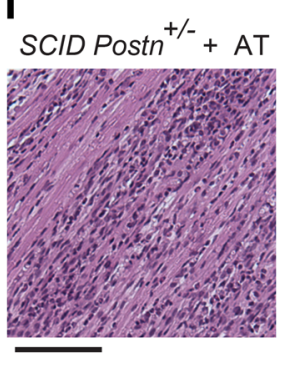

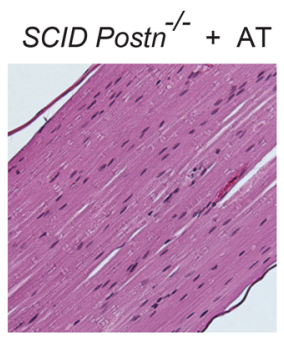

J

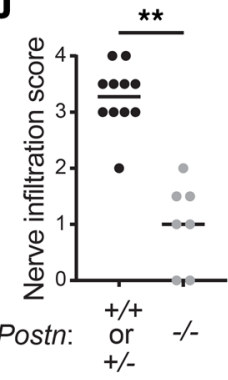

Figure 3. Postn deficiency protects against neuropathy. NOD.SCID Postn ${ }^{+/+}$, NOD.SCID Postn ${ }^{+/-}$, and NOD.SCID Postn ${ }^{-/-}$mice after AT with NOD. Aire CW/+ $^{-1}$ activated splenocytes. NOD.SCID Postn ${ }^{-1-}$ mice were compared with NOD.SCID Postn ${ }^{+/+}$and NOD.SCID Postn ${ }^{+/-}$mice. (A) Neuropathy incidence curve shows the onset of clinical symptoms. Dotted line represents the time point at which mice were used in experiments (10 weeks after AT). (B) Representative CMAP traces 10 weeks after AT. The CMAP of the NOD.SCID Postn ${ }^{+/-}$nerve had a smaller amplitude and was more dispersed than that of the NOD.SCID Postn $^{-1-}$ nerve (note the difference in the $y$-axis scale: 2 vs. $20 \mathrm{mV}$ ). (C) Peak amplitude, (D) conduction velocity, and (E) duration of CMAPs for mice in A. Each dot represents an individual mouse. (F) Representative images of semithin sections of sciatic nerves from NOD.SCID Post $n^{+/-}(n=8)$ and NOD.SCID Postn $^{-/-}(n=3)$ recipients of NOD. Aire ${ }^{C W /+}$ activated splenocytes. Original magnification, $\times 100$. (G) The number of myelinated axons $/ 1,000 \mu \mathrm{m}^{2}$ was counted in cross sections of tibial nerves from NOD.SCID Postn ${ }^{+-}$and NOD.SCID Postn ${ }^{-1-}$ AT recipients. (H) EM images. Demyelinated axons (indicated by asterisks) were observed in the NOD.SCID Postn ${ }^{+-}$nerve $(n=8)$, whereas the NOD.SCID Postn ${ }^{-/}(n=3)$ nerve looked normal. Scale bar: $2 \mu \mathrm{m}$. (I) H\&E staining of sciatic nerves. The NOD.SCID Postn ${ }^{+/-}$nerve was heavily infiltrated by hematoxylin-positive nuclei compared with the NOD.SCID Postn ${ }^{-/-}$nerve. Scale bar: $12 \mu \mathrm{m}$. (J) Infiltration scores were assigned as follows: $0=$ no infiltration; $1=1 \%-25 \%$ of the nerve was infiltrated; $2=26 \%-50 \%$ of the nerve was infiltrated; $3=51 \%-75 \%$ of the nerve was infiltrated; $4=76 \%-100 \%$ of the nerve was infiltrated. Each dot represents an individual mouse. ${ }^{*} P<0.05$ and ${ }^{* *} P<0.005$, by log-rank test (A), 2-tailed, unpaired $t$ test with Welch's correction (C-E and $\mathbf{G}$ ), or Fisher's exact test (J).

between POSTN and S100B, indicating that Schwann cells are the source of POSTN in human CIDP patients as well as in murine SAPP. As a complementary approach, we sought to determine whether a HSC line can be induced to express POSTN. It has been reported that Neuregulin 1 (NRG1) and TGF- $\beta$ can induce Postn expression in cultured rat Schwann cells (35). Further, Tgfb is highly expressed in the sciatic nerve of SAPP mice (Supplemental Figure 7), suggesting its presence at the site of inflammation. Moreover, infiltrating $\mathrm{CD}^{+} \mathrm{T}$ cells express high levels of $\mathrm{Tg} f b$ (data not shown). Given these data, we cultured immortalized HSCs in the presence of NRG1, TGF- $\beta$, or both NRG1 and TGF- $\beta$, and measured POSTN expression by qRT-PCR (Figure 2H). POSTN expression was increased by 3 -fold in HSCs treated with TGF- $\beta$ or TGF- $\beta$ and NRG1, but not in HSCs treated with NRG1 alone. These data indicate that TGF- $\beta$ induces POSTN expression in HSCs and support the notion that Schwann cells are the source of increased POSTN expression in CIDP.

Postn deficiency delays the onset of neuropathy. Mice homozygous for the Postn lacZ reporter allele also lack functional Postn expression, because the $\beta$-gal cassette replaces exons $4-10$ of the Postn gene (45). Thus, NOD.Postn ${ }^{\text {lacz/lacz }}$ mice are effectively Postn knockouts (hereafter referred to as NOD.Postn-/-). Although 
A
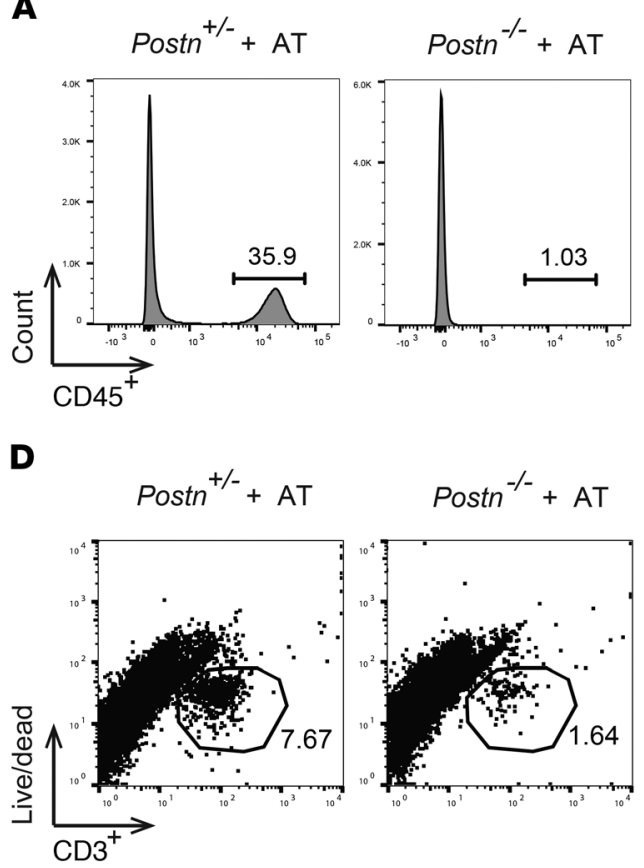

G

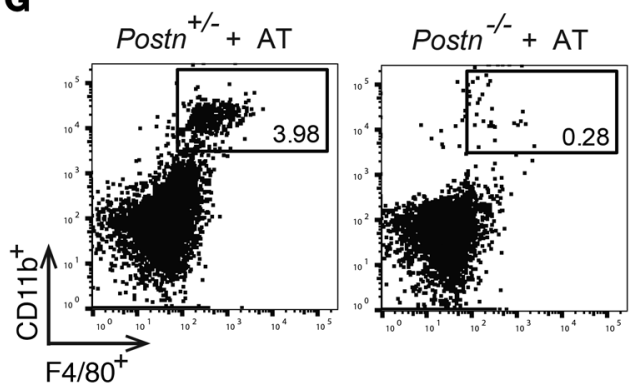

B

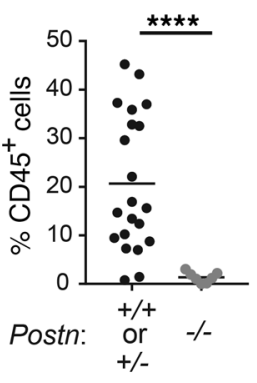

$\mathbf{E}$

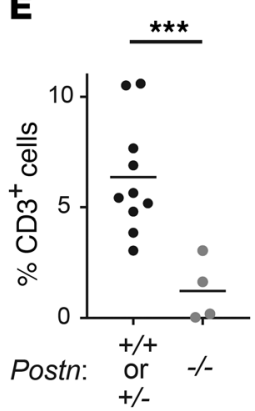

H

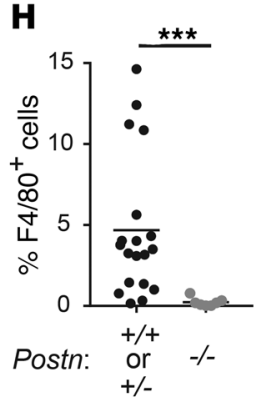

C

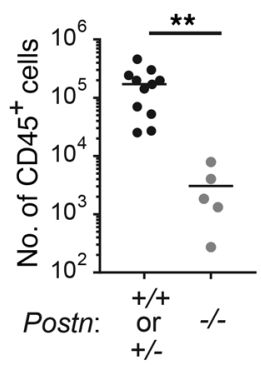

$\mathbf{F}$

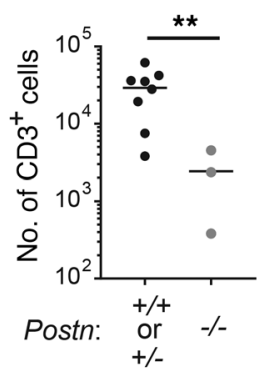

I

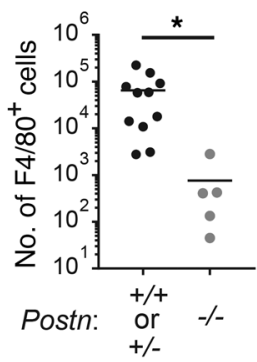

Figure 4. Postn-deficient mice have fewer infiltrating $T$ cells and macrophages.

Flow cytometry was used to evaluate the immune cell infiltrate of sciatic nerves from NOD.SCID Postn ${ }^{+/+}$, NOD.SCID Postn ${ }^{+/}$, and NOD.SCID Postn ${ }^{-/}$mice 10 weeks after AT with NOD. Aire ${ }^{\mathrm{CW} /+}$ activated splenocytes. (A) Representative histograms of CD45 staining. (B) Frequency and (C) total number of CD45 $5^{+}$cells. (D) Representative dot plots of $\mathrm{CD}^{+}$versus live/dead staining. (E) Frequency and (F) total number of $\mathrm{CD}^{+}$cells. (G) Representative flow plots of $\mathrm{F} 4 / 80^{+}$versus $\mathrm{CD}^{-1} \mathrm{~b}^{+}$ staining. (H) Frequency and (I) total number of $\mathrm{F} 4 / 8 \mathrm{O}^{+} \mathrm{CD} 11 \mathrm{~b}^{+}$cells. Each dot represents an individual mouse. ${ }^{*} P<0.05$, ${ }^{*} P<0.005$, ${ }^{* *} P<0.0005$, and ${ }^{* * *} P<$ 0.0001 , by 2 -tailed, unpaired $t$ test with Welch's correction.
Postn is expressed in the PNS during embryonic development, it has been reported that Postn-deficient mice have normal PNS function (35) demonstrating that Postn is dispensable for PNS development. Consistent with this, we saw no overt signs of PNS impairment in NOD.Postn ${ }^{-/}$mice when assessed clinically (Supplemental Figure 8A), electrophysiologically (Supplemental Figure 8, B-E), or anatomically (Supplemental Figure 8, F-H). Thus, genetic Postn deficiency in mice does not alter PNS structure or function or preclude the use of Postn-deficient mice to probe the role of Postn in PNS autoimmunity.

To undertake these studies, we used the AT model of inflammatory neuropathy, in which splenocytes from Postn-sufficient, neuropathic NOD.Aire ${ }^{G W /+}$ mice were transferred into Postndeficient NOD.SCID recipients (NOD.SCIDPostn ${ }^{-/}$). Reconstituted recipient mice were Postn deficient in non-T and -B cell types (including Schwann cells), since NOD.SCID Postn ${ }^{-/}$recipient mice lack $\mathrm{T}$ and B cells. Splenocyte transfer provoked neuropathy in more than $90 \%$ of Postn-sufficient (NOD.SCID Postn ${ }^{+/+}$or NOD. $S C I D$ Post $^{+/-}$) recipient mice by 12 weeks after transfer (Figure 3A). The incidence of neuropathy was not different between Postn

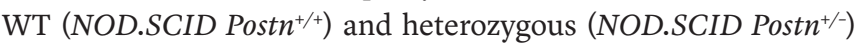
recipient mice (Supplemental Figure 9), suggesting that heterozygosity for the mutant Postn allele does not have a functional con- sequence. In contrast, we found that NOD.SCID Postn ${ }^{-/}$recipient mice were protected from neuropathy, since splenocyte transfer provoked neuropathy in only $40 \%$ of NOD.SCID Postn ${ }^{-/}$recipients by 12 weeks after transfer (Figure 3A). Thus, Postn deficiency in non-T and -B cells ameliorates clinical neuropathy.

To further characterize PNS disease in these mice, we performed electromyography (EMG) on recipients 10 weeks after AT, or earlier, if moderate or severe neuropathy (clinical score of 3) was noted. The 10-week time point was chosen, because most Postn-sufficient recipient mice develop neuropathy by this time. EMG revealed that Postn-sufficient recipients had significantly reduced compound muscle action potential (CMAP) peak amplitudes (Figure 3, B and C), slower conduction velocities (Figure 3D), and increased CMAP duration (Figure 3, B and E) compared with NOD.SCID Postn ${ }^{-1}$ recipient mice 10 weeks after AT. The combination of increased CMAP durations and reduced conduction velocities suggested that more axons were demyelinated in the Postn-sufficient recipient mice. To investigate this point, we examined sciatic nerves by light microscopy (semithin sections) and EM (thin sections) and found larger regions of demyelinated axons in Postn-sufficient recipients compared with Postndeficient recipients (Figure 3, $\mathrm{F}$ and $\mathrm{H}$ ). In order to quantify demyelination, we compared the density of myelinated axons in tibial 

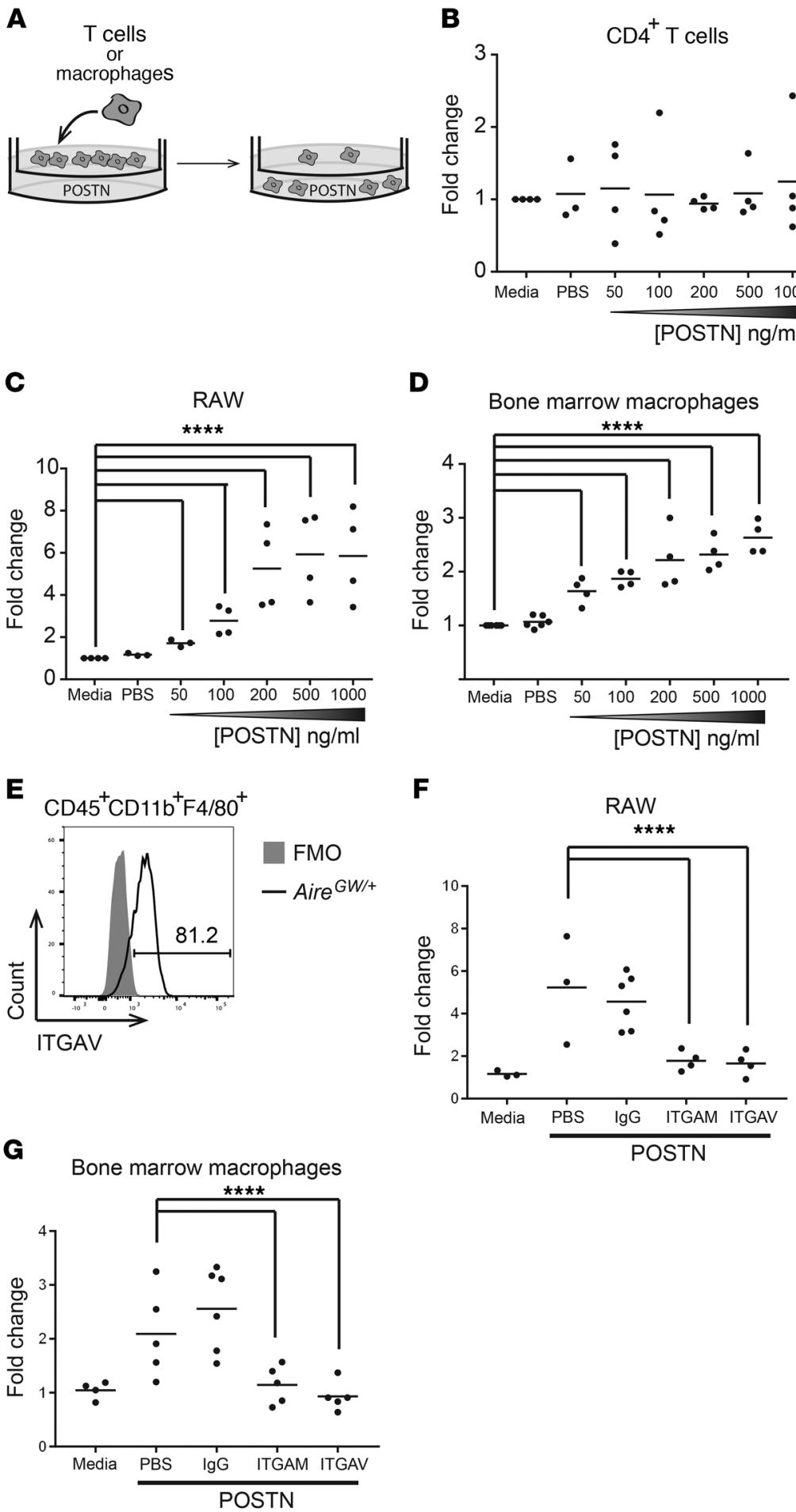

B

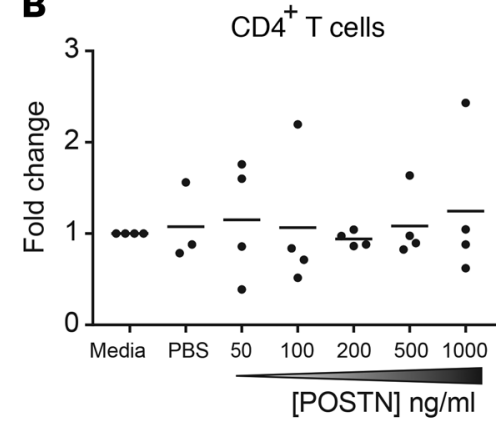

$\mathbf{F}$

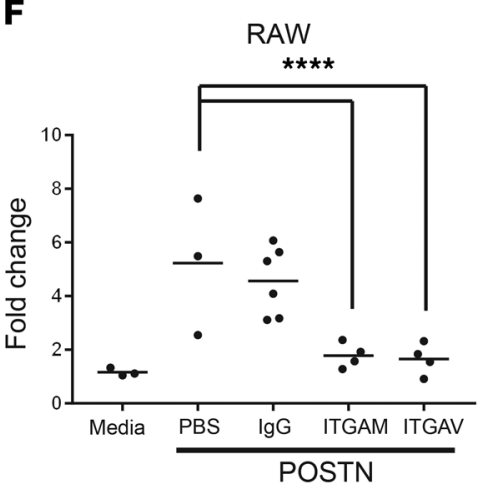

Figure 5. POSTN promotes macrophage chemotaxis via AM and AV integrins. (A) Transwell migration assay diagram. T cells or macrophages were placed in the upper chamber of a Transwell, and POSTN was placed in the lower chamber. Following a 4-hour (T cells) or 18-hour (macrophages) culture, the number of cells in the bottom chamber was enumerated. The concentration of POSTN was varied from 50 to 1,000 ng/ $\mathrm{ml}$. (B) $\mathrm{CD} 4^{+} \mathrm{T}$ cells isolated from the spleens of neuropathic NOD.Aire ${ }^{C W /+}$ mice were used in a Transwell migration assay as outlined in $\mathbf{A}$. Each dot represents an individual mouse. (C) RAW macrophages were used in a Transwell migration assay. Each dot represents an individual well. (D) Bone marrow was isolated from NOD. Aire $\mathrm{CW}_{\mathrm{W} / \mathrm{t}}$ neuropathic mice and grown in the presence of GM-CSF for 5 days to generate bone marrow-derived macrophages, which were used in a Transwell migration assay. Each dot represents an individual mouse. (E) Sciatic nerves from neuropathic NOD. Aire ${ }^{\mathrm{CW} /+}$ mice were digested and stained for flow cytometry. A representative histogram of ITCAV expression on CD45 ${ }^{+} \mathrm{CD} 11 \mathrm{~b}^{+} \mathrm{F} 4 / 80^{+}$ macrophages is shown. ITCAV expression was compared with a fluorescence-minus-one (FMO) control. The number represents the frequency of events within the gate. RAW cells $(\mathbf{F})$ or bone marrow-derived macrophages (G) were used in a Transwell migration assay with $100 \mathrm{ng} / \mathrm{ml}$ POSTN. IgG isotype control, anti-ITCAM (CD11b), or anti-ITCAV antibodies were included in the top chamber of the Transwell. All values are represented as the fold change compared with media alone. ${ }^{* * *} P<0.0001$. Statistical analysis was performed in $\mathrm{R}$ using the $\operatorname{Im}($ ) function, where parameters were fit to each mouse as well as the difference in treatment. $P$ values were calculated by comparing the fit of the full model to a model without a term for the difference in treatment and adjusted using Bonferroni's correction. nerve cross sections. As expected, the number of myelinated axons/1,000 $\mu \mathrm{m}^{2}$ was significantly lower in Postn-sufficient AT recipient mice than in Postn-deficient mice (Figure 3G). In addition to demyelination, other factors such as edema and immune cell infiltration may contribute to the decreased myelinated nerve density observed in neuropathic animals. Indeed, the regions of demyelinated axons contained many cells that were not surrounded by a basal lamina and had the ultrastructural features of macrophages and lymphocytes. To investigate this issue further, we stained paraffin sections of sciatic nerves with $\mathrm{H} \& \mathrm{E}$ and observed decreased cellular infiltration of the sciatic nerve in NOD.SCID Postn ${ }^{-/}$recipients compared with Postn-sufficient recipients (Figure 3, I and $\mathrm{J}$ ).

We next used flow cytometry to determine the cellular composition of sciatic nerves in reconstituted Postn-sufficient versus Postn-deficient recipient mice. We found decreased frequency and fewer absolute numbers of infiltrating $\mathrm{CD} 45^{+}$hematopoietic cells in sciatic nerves of NOD.SCID Postn ${ }^{-/}$recipients compared with Postn-sufficient recipients (Figure 4, A-C), confirming decreased immune cell infiltration with Postn deficiency. To deter- 
A

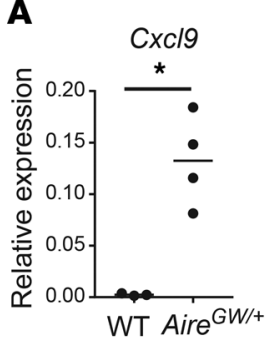

F

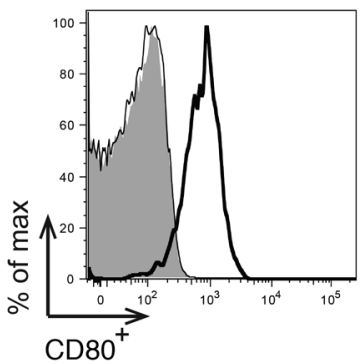

B

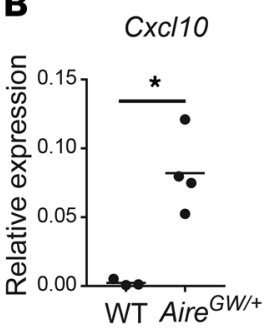

G

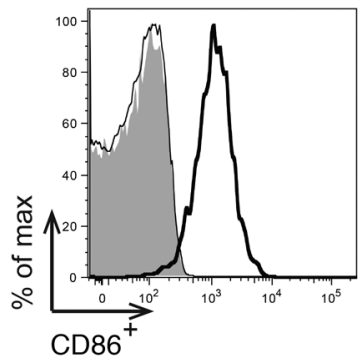

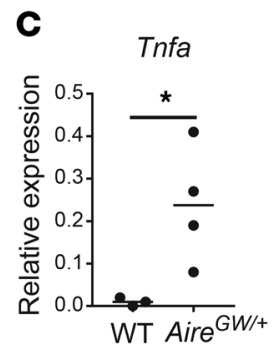

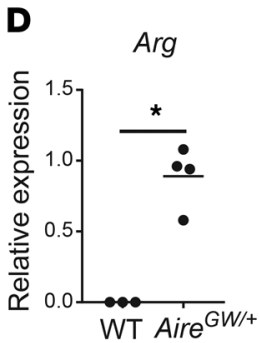

H

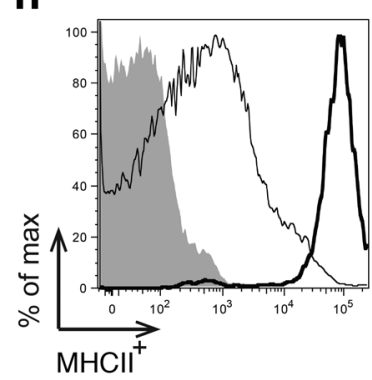

K
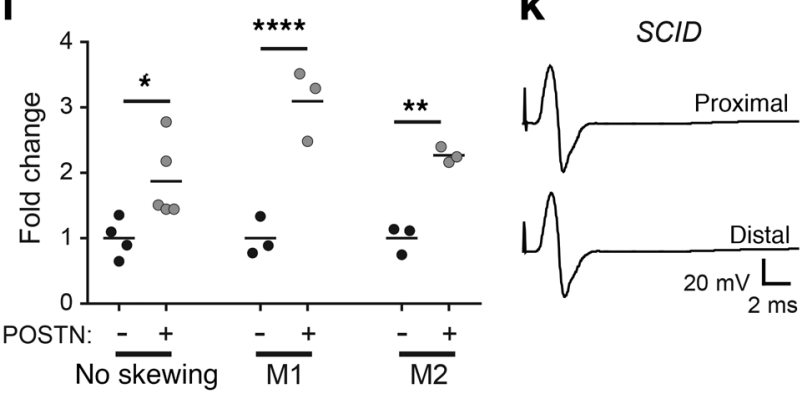

$S C I D+A T+$ vehicle
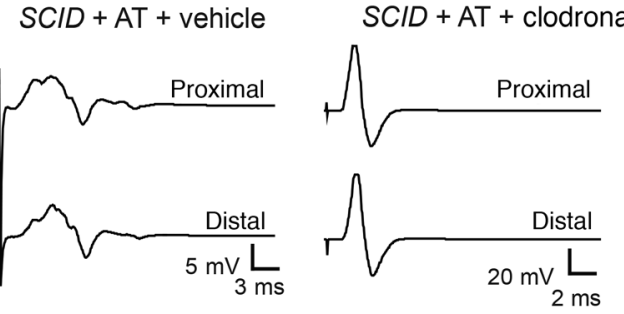

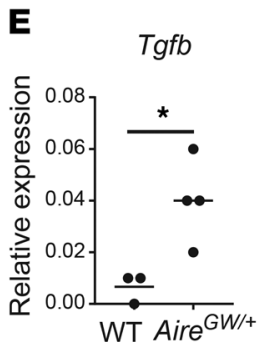

WT nonmacrophage - Aire ${ }^{G W /+}$ nonmacrophage - Aire ${ }^{G W /+}$ macrophage

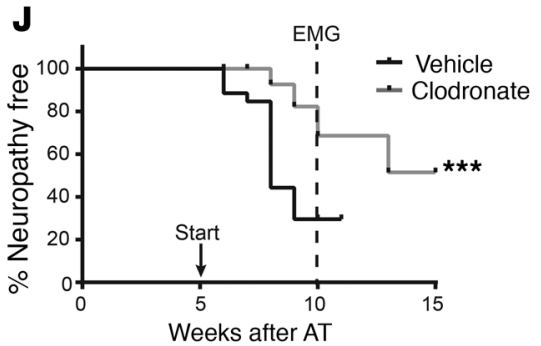

$\mathbf{L}$

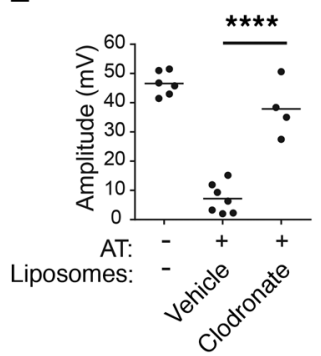

M

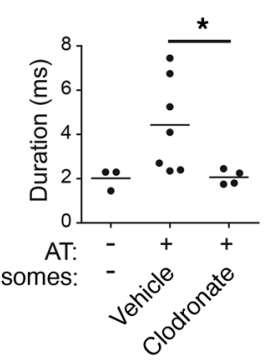

Figure 6. Macrophages promote peripheral neuropathy. (A-C) RNA was isolated from the sciatic nerves of NOD.WT (WT) mice and from CD3-CD11b+ enriched macrophages from the sciatic nerves of NOD. Aire ${ }^{C W /+}$ neuropathic mice. Cxclg (A), Cxcl10 (B), Tnfa (C), Arg (D), and Tgfb (E) expression relative to cyclophilin was measured by qRT-PCR. (F-H) Sciatic nerves from NOD or NOD.Aire ${ }^{C W /+}$ neuropathic mice were digested and stained for flow cytometric analysis. Representative histograms showing $\mathrm{CD} \mathrm{O}^{+}(\mathbf{F}), \mathrm{CD} \mathrm{6}^{+}(\mathbf{G})$, and $\mathrm{MHCII}^{+}(\mathbf{H})$ expression on macrophages and nonmacrophages from NOD or NOD.Aire ${ }^{\mathrm{CW} /+}$ neuropathic mice. (I) Macrophages were skewed toward an M1 or M2 phenotype prior to inclusion in a Transwell migration assay; $100 \mathrm{ng} / \mathrm{ml}$ P0STN was used in the bottom chamber. All values are represented as the fold change compared with media alone. (J-N) NOD.SCID recipients of NOD.Aire ${ }^{\mathrm{CW} /+}$ splenocytes were treated with vehicle or clodronate-containing liposomes weekly starting 5 weeks (arrow) after AT; nerve conductions were done at 10 weeks (dotted line in J). (J) Neuropathy incidence curve shows that fewer clodronate-treated mice developed neuropathy. (K) Representative EMG CMAPs from untreated NOD. SCID mice as well as post-AT NOD.SCID mice treated with vehicle or clodronate liposomes (note the difference in the scale of the $y$ axis: 5 vs. 20 mV). Compared with vehicle, clodronate significantly reduced the CMAP peak amplitudes (L), CMAP durations (M), and conduction velocities (N). Each dot represents an individual mouse. ${ }^{*} P<0.05$, ${ }^{* *} P<0.005$, ${ }^{* *} P<0.0005$, and ${ }^{* * * *} P<0.0001$, by 2-tailed $t$ test with Welch's correction (A-E), log-rank test (J), or ordinary 1-way ANOVA with Tukey's post hoc test for multiple comparisons (L-N). max, maximum.

mine whether Postn deficiency alters the cellular composition of immune cell infiltration, we compared the frequencies of $\mathrm{CD}^{+} \mathrm{T}$ cells and $\mathrm{F} 4 / 80^{+}$macrophages within the nerves. We previously reported that $\mathrm{T}$ cells and macrophages account for the majority of infiltrating cells in neuropathic NOD.Aire ${ }^{\mathrm{GW} / \mathrm{+}}$ mice (7). Likewise, immunohistochemical staining of sciatic nerve sections from reconstituted, neuropathic NOD.SCID recipients of transferred NOD.Aire ${ }^{G W /+}$ splenocytes showed increased $\mathrm{CD}^{+} \mathrm{T}$ cells and $\mathrm{F} 4 / 80^{+}$macrophages (Supplemental Figure 10). The frequency and absolute numbers of both $\mathrm{CD}^{+} \mathrm{T}$ cells (Figure 4, D-F) and 
$\mathrm{CD}_{11 \mathrm{~b}} \mathrm{~F}^{4} / 80^{+}$macrophages (Figure $4, \mathrm{G}-\mathrm{I}$ ) were reduced in reconstituted NOD.SCID Postn ${ }^{-/}$recipient mice compared with their Postn-sufficient counterparts. Thus, we conclude that neuropathy resistance in Postn-deficient recipient mice is associated with less demyelination and decreased immune cell infiltration.

POSTN promotes macrophage chemotaxis. Reduced $\mathrm{T}$ cell and macrophage numbers in the sciatic nerve of Postn-deficient AT recipient mice could be the result of reduced proliferation, reduced $\mathrm{T}$ cell activation, or increased cell death. If Postn deficiency impacts $\mathrm{T}$ cell or macrophage proliferation or death, one would expect to see fewer $\mathrm{T}$ cells and macrophages in the spleens of Postn-deficient mice. However, we observed no difference in the frequency or total number of $\mathrm{CD}^{+}, \mathrm{CD}^{+}$, or $\mathrm{CD}^{+} \mathrm{T}$ cells or $\mathrm{CD}_{11} \mathrm{~b}^{+}$macrophages (Supplemental Figure 11). Additionally, the frequency and number of $\mathrm{Ki}^{+} \mathrm{T}$ cells (Supplemental Figure 12, $\mathrm{A}-\mathrm{C}$ ) and macrophages (Supplemental Figure 12, D-F) were similar in NOD.Postn ${ }^{+/+}$and NOD.Postn ${ }^{-/-}$mice, suggesting that Postn deficiency does not affect $\mathrm{T}$ cell or macrophage proliferation. Likewise, we observed no difference in the frequency or number of effector/memory $\left(\mathrm{CD} 44^{+} \mathrm{CD} 62 \mathrm{~L}^{-}\right) \mathrm{CD}^{+} \mathrm{T}$ cells in Postndeficient mice compared with Postn-sufficient mice (Supplemental Figure 12, G-K). Finally, we detected no difference in the expression of the apoptotic regulator $\mathrm{Bcl} 2$ in macrophages treated in vitro with POSTN (Supplemental Figure 13). Taken together, these data suggest that reduced numbers of $\mathrm{T}$ cells and macrophages in the sciatic nerves of Postn-deficient AT recipients cannot be explained by altered immune cell proliferation, activation, or death.

Another possible explanation for decreased $\mathrm{T}$ cell and macrophage nerve infiltration in Postn-deficient mice is that POSTN normally promotes the migration of these immune cell types into the PNS. We used a Transwell system (Figure 5A) to test the ability of $\mathrm{CD} 4^{+} \mathrm{T}$ cells or macrophages to migrate toward increasing concentrations of POSTN protein. POSTN failed to induce the migration of $\mathrm{CD}^{+} \mathrm{T}$ cells that were isolated from the spleens of neuropathic NOD.Aire ${ }^{G W /+}$ mice, regardless of the concentration of POSTN (Figure 5B). However, the migration of RAW cells, a macrophage-derived cell line, was increased in response to POSTN in a dose-dependent manner (Figure 5C). We observed similar results when NOD.Aire ${ }^{G W /+}$ bone marrow-derived macrophages were used in place of RAW cells (Figure 5D). Known POSTN receptors include the integrins ITGAV (42) and ITGAM (CD11b) (41), which have been implicated in mediating various POSTN-dependent effects, and macrophages in infiltrated sciatic nerves express both CD11b (also known as ITGAM) (Figure 4G) and ITGAV (Figure 5E). Inclusion of blocking antibodies specific to CD11b/ITGAM or ITGAV in the upper chamber of the Transwell prevented RAW cells and NOD.Aire ${ }^{G W /+}$ bone marrow-derived macrophages from migrating across the membrane, whereas IgG isotype control antibodies did not (Figure 5, F and G). These data demonstrate that POSTN is directly chemotactic to macrophages but not T cells and that this response requires ITGAV and ITGAM.

Macrophages promote the development of PNS autoimmunity. While macrophages are a predominant cell type in the immune cell infiltrate of neuropathic nerves, their role in the development of autoimmune peripheral neuropathy is unclear. To address this question, we first assessed the expression of M1- and M2associated markers. We isolated macrophages from the sciatic nerves of neuropathic NOD.Aire ${ }^{G W /+}$ mice and measured the expression of M1- and M2-associated markers by qRT-PCR. Since NOD.WT sciatic nerves have few resident macrophages, we used whole NOD.WT nerve tissue as a comparison. No significant difference was seen in the expression of the M1-associated markers Il12, iNos, or Cd86 or the M2-associated markers Il1O, Fizz, Mrc, or Ym1 (Supplemental Figure 14); however, we detected a significant increase in the M1-associated markers Cxcxl9, Cxcl10, and Tnfa and the M2-associated markers Arg and Tgfb (Figure 6, A-E). Additionally, we used flow cytometry to measure the activation markers CD80, CD86, and MHCII on nerve-infiltrating $\mathrm{CD} 11 \mathrm{~b}^{+} \mathrm{F} 4 / 80^{+}$macrophages from the sciatic nerves of neuropathic NOD.Aire ${ }^{G W /+}$ mice. Compared with CD11b-F4/80- nonmacrophage cells, CD11b ${ }^{+} \mathrm{F} 4 / 80^{+}$macrophages expressed more CD80, CD86, and MHCII (Figure 6, $\mathrm{F}-\mathrm{H})$. CD11b-F4/80- nonmacrophage cells from sciatic nerves of NOD.Aire $\mathrm{GW}^{\mathrm{G} / \mathrm{+}}$ mice expressed an intermediate amount of MHCII (Figure 6H), which may reflect an upregulation of MHCII expression by other cell types such as Schwann cells (51-53). These data suggest that nerve-infiltrating macrophages are heterogeneous, expressing both M1- and M2-associated markers, and express markers of activation.

Since nerve-infiltrating macrophages express both M1- and M2-associated markers, we wondered whether POSTN attracts macrophages of a specific phenotype. POSTN has been suggested to recruit M2 antiinflammatory macrophages (54), but whether POSTN also recruits the M1 macrophage subtype is unclear. To test this, macrophages were skewed toward an M1 or M2 phenotype prior to inclusion in a Transwell migration assay. Unskewed, and M1- and M2-skewed macrophages all migrated to a similar degree in response to POSTN (Figure 6I), indicating that POSTN is a chemotactic factor for both M1 and M2 macrophages. It has also been reported that POSTN can regulate the expression of markers of M1 and M2 polarization (54). To determine whether POSTN alters macrophage polarization, we skewed macrophages toward an M1 or M2 phenotype in the presence or absence of POSTN and measured the expression of M1 and M2 markers by qRT-PCR. POSTN did not change the expression of the M1-associated markers Tnfa and iNos or the M2associated marker Arg (Supplemental Figure 15). Thus, POSTN does not impact macrophage polarization in vitro.

Next, to determine whether macrophages contribute to SAPP, we treated NOD.Aire ${ }^{G W /+}$ mice between 7 and 14 weeks of age with clodronate liposomes to deplete phagocytic cells. Uptake of clodronate liposomes by phagocytes induces their apoptosis, and clodronate liposomes have previously been used to deplete macrophages in autoimmune mouse models (55-58). As expected, fewer $\mathrm{F} 4 / 8 \mathrm{O}^{+} \mathrm{CD} 11 \mathrm{~b}^{+}$macrophages were noted in the spleens of mice 3 days after treatment with clodronate compared with vehicle control liposome numbers (Supplemental Figure 16). Clodronate treatment was associated with a significant delay in the onset of peripheral neuropathy in NOD.Aire ${ }^{G W /+}$ mice compared with vehicle liposome treatment (Supplemental Figure 17A). Flow cytometric analysis of sciatic nerves from 21-week-old NOD.Aire ${ }^{G W /+}$ mice demonstrated decreased frequency and absolute numbers of $\mathrm{CD} 11 \mathrm{~b}^{+} \mathrm{F} 4 / 80^{+}$macrophages in the sciatic nerves of clodronate liposome-treated mice. Thus, clodronate treatment protects 
against SAPP through macrophage depletion (Supplemental Figure 17, B and C). In parallel, we investigated whether macrophage depletion was similarly protective in the AT model of inflammatory neuropathy. We administered clodronate or vehicle liposomes to reconstituted NOD.SCID recipients from 5 weeks after transfer until the mice developed neuropathy or were used in further experiments. The onset of clinical neuropathy in reconstituted recipients was significantly delayed with clodronate compared with vehicle liposome treatment (Figure 6J). Moreover, nerve conductions performed 5 weeks after administering clodronate (10 weeks after administration of splenocytes) (Figure 6, K-N) showed increased CMAP peak amplitudes (Figure 6L), decreased CMAP durations (Figure 6M), and faster nerve conduction velocities (Figure 6N) with clodronate treatment. Thus, in the AT model, clodronate-mediated depletion of macrophages protects against the development of demyelinating neuropathy, which suggests a pathogenic role for macrophages in inflammatory neuropathy.

\section{Discussion}

The development of tissue-specific autoimmunity involves a complex interplay between autoreactive immune cells and the target tissue. Whether the PNS regulates disease progression in autoimmune neuropathies, however, has not been documented. We show here that Schwann cells participate in the immune response by upregulating the ECM protein POSTN. Postn expression was increased in multiple mouse models of CIDP, suggesting that this may be a generalizable finding in inflammatory neuropathies. The absence of Postn delayed the onset of disease and reduced the severity of neuropathy. Postn deficiency also resulted in improved nerve function by EMG and reduced immune cell infiltration into nerves. Together, these findings suggest that Schwann cell-derived POSTN promotes PNS autoimmunity by enhancing immune cell infiltration (Supplemental Figure 18).

Postn is upregulated in various diseases and injuries (59) including cancer (60), myocardial infarction (61), asthma (62), skin inflammation (21), glomerulonephritis (22), and colitis (43). TGF- $\beta$ or neuregulin/ERBB3 signaling $(35,63)$ can induce Postn expression in Schwann cells during development; additionally, Postn expression can also be induced by IL-4 and IL-13 in keratinocytes during allergic skin inflammation (21) and by TNF- $\alpha$ and IL-17 during liver fibrosis (64). POSTN signals through ITGAV/ B3 (21, 42), ITGAV/B5 (42), and ITGAM (eosinophils) (41) and results in the phosphorylation of focal adhesion kinase (FAK) (65) and AKT (65) in neurons and the activation of $\mathrm{NF}-\mathrm{\kappa B}(21,43)$ in keratinocytes and colon. POSTN regulates various cytokines and chemokines including TNF- $\alpha$ in skin inflammation and following spinal cord injury $(21,23)$; IL-1A, granulocyte-macrophageCSF (GM-CSF), and the chemokine ligand CCL17 in allergic skin inflammation and glioblastoma $(21,54)$; RANTES (CCL5) in kidney and nasal epithelia $(22,66)$; and CCL2, CCL4, CCL7, and CXCL2 in pulmonary fibrosis (20). Additionally, POSTN has been shown to promote immune cell recruitment to sites of inflammation in spinal cord injury and glioblastoma $(20,23,54)$. We observed a similar effect of POSTN on the recruitment of immune cells to the sciatic nerve during SAPP.

While both $\mathrm{T}$ cell and macrophage numbers were reduced in peripheral nerves of Postn-deficient mice, only macrophages responded to POSTN in an in vitro Transwell migration assay. This finding suggests that POSTN primarily promotes macrophage chemotaxis and does not directly promote $\mathrm{T}$ cell chemotaxis. Instead, POSTN may promote $\mathrm{T}$ cell influx indirectly through the recruitment of macrophages that in turn express $\mathrm{T}$ cell chemoattractants. This notion is consistent with our observation (Figure 6, A and B) that nerve-infiltrating macrophages express the T cell chemoattractants $\mathrm{Cxcl} 9$ and $\mathrm{Cxcl10}$. Additionally, POSTN has been reported to regulate other $\mathrm{T}$ cell chemoattractants including RANTES $(22,66)$, which is secreted by macrophages during EAN (9), and the chemokine ligand CCL17 (21). Thus, we propose that a positive feedback loop exists between $\mathrm{T}$ cells and macrophages. This cycle is probably initiated by PNS-specific $\mathrm{CD} 4^{+} \mathrm{T}$ cells, given our data that $\mathrm{CD} 4^{+} \mathrm{T}$ cells are sufficient to incite SAPP in immunodeficient mice (7). These early infiltrating T cells produce TGF- $\beta$, which induces Schwann cells to express Postn. By binding to ITGAM and ITGAV on macrophages, POSTN attracts macrophages into the endoneurium. This amplifies a positive feedback loop between macrophages and $\mathrm{T}$ cells through increased macrophage expression of the $\mathrm{T}$ cell chemoattractants Cxcl9 and Cxcl10 (Supplemental Figure 18).

In mouse and rat models of nerve injury, macrophages are recruited 3-5 days after injury (67). In this setting, macrophages are important in clearing myelin debris and participate in nerve regeneration by secreting growth-promoting factors and cytokines that act upon Schwann cells and neurites (68). Given this reparative role for macrophages during axotomy, the promotion by Postn of macrophage infiltration may have both proinflammatory and reparative effects. Further, it is also possible that Postndependent macrophage infiltration may even be antiinflammatory, since nerve-infiltrating macrophages express some immunosuppressive M2-associated markers, and data from our group and others show that Postn can promote M2 macrophage chemotaxis (54). Our results indicate that macrophages overall were proinflammatory during PNS autoimmunity, since macrophage depletion delayed the onset of disease. Nevertheless, it is important to acknowledge that the macrophage population is likely to be heterogeneous (69), since both M1- and M2-associated markers were expressed (Figure 6, A-E), and that a subset of PNSinfiltrating macrophages may ameliorate disease. Further delineation of macrophage subsets and their function in SAPP will be the subject of future studies.

In addition to SAPP and axotomy, other disease states, including some hereditary neuropathies, are also associated with macrophage infiltration into the PNS (70). At present, it is not clear whether the same chemotactic factors mediate macrophage influx in each of these conditions. Multiple factors, such as CCR2-CCL2 interactions, have been implicated in macrophage recruitment after nerve injury (71). CCR2 is expressed on infiltrating macrophages, and CCL2 (also known as MCP) secretion by Schwann cells recruits macrophages into the injured nerve (72-74). Additionally, MIP1A and IL-1 $\beta$ also play important roles in macrophage chemotaxis with axotomy (72). Whether POSTN also plays a role in macrophage infiltration with hereditary neuropathies and whether CCR2-CCL2, MIP1A, and IL-1 $\beta$ might promote macrophage infiltration during autoimmune peripheral neuropathy await further investigation.

In the CNS, Postn is expressed by several glial cell types including astrocytes (65) and pericytes (23) following injury. Postn 
promotes scar formation, regulates blood-brain barrier permeability, and induces proinflammatory molecules such as MMP9, leading to impaired functional recovery following spinal cord injury (23) and increased pathology following subarachnoid hemorrhage (75). In contrast to the CNS, we did not observe increased Postn expression following PNS injury. The regenerative capacity of the PNS is markedly better than that of the CNS; however, the underlying reasons for this difference in regenerative capacity are poorly understood. Since Postn deficiency improves recovery following CNS injury, we speculate that a lack of Postn upregulation following PNS injury may partially account for the improved ability of the PNS to repair itself.

In preclinical trials, POSTN inhibitors have shown promise as cancer therapeutics, and both antibody-based and aptamer POSTN inhibitors are actively being pursued $(76,77)$. Our finding that POSTN is induced in mouse models of inflammatory neuropathy and required for macrophage-mediated disease acceleration suggests that POSTN inhibition may be a potential immunotherapeutic strategy for inflammatory neuropathies. Since POSTN recruits macrophages to the PNS through interaction with ITGAV, targeting ITGAV is another potential strategy implicated by our results. Currently, several antibody and small-molecule inhibitors of ITGAV are in phase I and phase II clinical trials for the treatment of cancer $(78,79)$. Our results provide a basis for the development of these molecules as novel therapeutics for inflammatory neuropathies.

\section{Methods}

\section{Animals}

Strains and housing. Mice were housed in a specific pathogen-free facility at the UNC-CH. NOD.Aire ${ }^{\mathrm{GW} / \mathrm{+}}$ and myelin protein zero T cell receptor-Tg (NOD.POT) mice were generated as previously described $(44,80)$. NOD.POT Tcr-Tg mice were a gift of Jeffrey Bluestone (UCSF, San Francisco, California, USA). NOD.SCID mice were purchased from The Jackson Laboratory. B6.Postn ${ }^{\text {lacz/+ }}$ mice (45) were purchased from The Jackson Laboratory and were backcrossed onto the nonobese diabetic (NOD) background for 8 generations using speed congenics (80). Genome scanning to select breeders of highest NOD strain origin was performed by JAX services using a 150 SNP panel that was polymorphic between NOD and C57BL/6. NOD. Postn ${ }^{\text {lacz/+ }}$ were then crossed with NOD.SCID mice to generate NOD.Post $n^{\text {lacz/+ }}$ SCID and NOD.Postn $n^{\text {lacz/lacZ }}$ SCID (also referred to as NOD.Postn ${ }^{-/}$SCID) mice.

AT model. Forty-eight-well plates were coated with anti-CD3 (eBioscience, clone 145-2C11; $1 \mu \mathrm{g} / \mathrm{ml}$ in PBS) and anti-CD28 (BD Pharmingen, clone $37.51 ; 1 \mu \mathrm{g} / \mathrm{ml}$ in PBS) overnight at $4^{\circ} \mathrm{C}$ or for at least 2 hours at $37^{\circ} \mathrm{C}$ and washed with PBS. Splenocytes from neuropathic NOD.Aire ${ }^{G W /+}$ or NOD.POT mice were plated at a density of $10^{6}$ (NOD. Aire ${ }^{\mathrm{GW} /+}$ ) or $5 \times 10^{5}$ (POT) cells/well and cultured for 96 to 120 hours in DMEM supplemented with 10\% FBS, 10 mM HEPES buffer (NOD.POT mice only), penicillin-streptomycin (100 U/ml, Gibco, Thermo Fisher Scientific), and 2-mercaptoethanol $(55 \mu \mathrm{M})$. Activated splenocytes (1 $\times 10^{6}$ ) were harvested, washed once with sterile PBS, and adoptively transferred in sterile PBS using retro-orbital injection into immunodeficient NOD.SCID recipients sedated with $2 \%$ isoflurane in oxygen.

Evaluation of SAPP. Mice were evaluated weekly (spontaneous model) or every other day (AT model) for clinical signs of neuropathy, which was scored as follows: 0 = no signs of neuropathy present; $1=$ mild hind limb weakness; 2 = pronounced bilateral hind limb weakness; 3 = reduced or absent ability to grip cage grating; and $4=$ moribund. For the neuropathy incidence curve, mice were considered neuropathic when they reached a score of 2 in order to limit the effect of false positives. SAPP was confirmed by electromyography, which was performed as previously described $(81,82)$.

Surgery. Mice were anesthetized with isoflurane and $100 \mathrm{mg} / \mathrm{kg}$ ketamine $/ 10 \mathrm{mg} / \mathrm{kg}$ xylazine or $250 \mathrm{mg} / \mathrm{kg}$ avertin. Fur was removed from the surgical area with depilatory cream and cleaned with alcohol wipes. The sciatic nerve was exposed and transected at the sciatic notch. The contralateral nerve was exposed but left intact (mock). The wound was sealed with surgical glue. Meloxicam $(10 \mathrm{mg} / \mathrm{kg})$ was administered at the time of surgery, and $2 \mathrm{mg} / \mathrm{kg}$ meloxicam was administered daily following surgery for pain management. Three days after nerve transection surgery, nerves were harvested, frozen in liquid nitrogen, and stored at $-80^{\circ} \mathrm{C}$ until use. RNA was extracted from transected and mock nerves, and Postn levels were measured by qRT-PCR.

\section{Flow cytometry}

Nerve digestion. Nerves were chopped into segments with a razor blade and digested in PBS containing $1 \mathrm{mg} / \mathrm{ml}$ collagenase and $1 \% \mathrm{FBS}$ at $37^{\circ} \mathrm{C}$ for 30 minutes with periodic agitation. Part way through the digestion, the sample was triturated with a 20-gauge needle to aid in tissue disruption. Dissociated nerves were filtered through a $40-\mu \mathrm{m}$ mesh and washed prior to use in experiments.

Antibodies. CD3 (clone 145-2C11), CD4 (clone RM4-5), CD8 (clone 53-6.7), F4/80 (clone BM8), and CD45 (clone 30F11) antibodies were purchased from eBioscience. CD11b (clone M1/70) was purchased from BioLegend. Live/Dead Fixable Yellow Dye (Life Technologies, Thermo Fisher Scientific) was used to exclude dead cells. Rabbit anti-p75 (1:500) was purchased from Abcam, and DyLight 488-conjugated goat antirabbit IgG (1:500) was purchased from Thermo Fisher Scientific.

Staining. Single-cell suspensions were washed twice with FACS buffer. Fc receptors were blocked by incubating cells with supernatant from a 2.4G2 hybridoma for 10 minutes. Cells were stained for 30 minutes on ice and washed prior to analysis.

Analysis. Samples were run on a Beckman Coulter CyAn ADP or BD LSR II flow cytometer and analyzed using FlowJo software. A BD FACSAria II was used for sorted samples.

\section{Immunostaining}

Mouse tissues. Nerves from NOD.Aire ${ }^{\mathrm{GW} / \mathrm{+}}$ and NOD.WT mice were embedded in OCT and frozen at $-20^{\circ} \mathrm{C}$ for 2 hours prior to storage at $-80^{\circ} \mathrm{C}$. Sciatic nerve cross sections or longitudinal sections $(6-\mu \mathrm{m})$ were fixed with cold acetone, blocked with $2.5 \%$ goat serum, and stained with $10 \mu \mathrm{g} / \mathrm{ml}$ anti-periostin (Abcam), anti-F4/80 (eBiocience), and anti-CD3 (BioLegend) for 2 hours. After washing with PBS containing $0.1 \%$ Tween (PBST), sections were incubated with anti-rat-HRP or anti-rabbit-HRP (VECTOR Laboratories) antibody for 30 minutes and developed in DAB solution until the desired stain intensity was achieved. All sections were processed in parallel, including the same length of time in the DAB solution. Samples were imaged on a Nikon Eclipse 80i wide-field microscope.

Humantissues. Slide-mounted cryosections of sciaticnerves from deidentified CIDP patients were air dried and fixed with $2 \%$ paraformaldehyde (PFA), washedin PBS, and permeabilized with $0.2 \%$ Triton 
X-100. After blocking with 5\% goat serum, sections were washed in PBS and incubated with 1:50 rabbit polyclonal anti-human POSTN (Abcam, catalog ab14041) and 1:200 mouse monoclonal anti-human S100B (Atlas, catalog AMAb91038, clone CL2720) for 18 hours at $4^{\circ} \mathrm{C}$. Sections were washed with PBS and incubated with 1:200 Alexa Fluor 594-labeled goat polyclonal anti-rabbit secondary antibody (Invitrogen, Thermo Fisher Scientific, catalog A11037) and 1:200 Alexa Fluor 488-labeled goat polyclonal antimouse secondary antibody (Invitrogen, catalog A11001) at room temperature for 1 hour. After incubation with a secondary antibody, slides were washed with PBS and coverslipped using antifade medium containing 4',6-diamidino-2-phenylindole (Prolong Gold, Invitrogen, Thermo Fisher Scientific, catalog P36931) and photographed using a Zeiss 800 confocal microscope.

\section{Transwell migration assay}

$T$ cells. Chemotaxis was measured using Transwell assays as described previously (83). Migration of CD $4^{+} \mathrm{T}$ cells was measured using a $24-$ well Transwell plate (Corning Life Sciences) with $6.5-\mathrm{mm}$ polycarbonate filters and $5-\mu \mathrm{m}$ pores. $\mathrm{CD} 4^{+} \mathrm{T}$ cells from neuropathic NOD.Aire ${ }^{\mathrm{GW} /+}$ spleens were purified using the Magnisort $\mathrm{CD} 4^{+} \mathrm{T}$ cell Enrichment Kit (Invitrogen, Thermo Fisher Scientific). Two million CD4 ${ }^{+} \mathrm{T}$ cells were resuspended in Transwell media (100 $\mu$ RPMI 1640 medium with $0.1 \%$ fatty acid-free BSA [Sigma-Aldrich], $100 \mathrm{U} / \mathrm{ml}$ penicillin G, $2 \mathrm{mM}$ L-glutamine, and $25 \mathrm{mM}$ HEPES buffer). Cells were placed on the Transwell inserts. Media $(600 \mu \mathrm{l})$, media plus PBS, or media plus 50-1,000 $\mathrm{ng} / \mathrm{ml}$ POSTN (R\&D Systems) were added to the lower chambers. Migration was performed for 4 hours at $37^{\circ} \mathrm{C}$ and $5 \% \mathrm{CO}_{2}$. Migrated cells were counted with a hemocytometer.

Macrophages. Bone marrow was flushed out of the femurs and tibiae of NOD.Aire ${ }^{G W /+}$ mice using PBS and then cultured in DMEM containing 10\% FBS, penicillin-streptomycin, and 10\% L cell supernatant for 5 days prior to use. RAW 264.7 cells or NOD.Aire ${ }^{G W /+}$ bone marrowderived macrophageswere cultured in DMEMwith10\% FBSina 24-well plate. Cells were washed 3 times with PBS, resuspended in DMEM without FBS, and seeded at $1 \times 10^{6}$ cells $/ \mathrm{ml}$ into the upper chamber in a total volume of $250 \mu \mathrm{l}$. Transwell inserts with $8-\mu \mathrm{m}$ pores (Corning) were used. Recombinant POSTN (R\&D Systems) was added to the lower chamber at 50,100, 200, 500, and 1,000 $\mathrm{ng} / \mathrm{ml}$ in DMEM with $5 \%$ FBS. Anti-CD11b (Thermo Fisher Scientific) and anti-CD51 (ITGAV) (BD) antibodies were added into the upper chamber at a concentration of $10 \mu \mathrm{g} / \mathrm{ml}$. For experiments comparing M1 and M2 macrophages, 200,000 bone marrow-derived macrophages were seeded in the upper chamber of the Transwell in a total volume of $350 \mu$ l. Macrophages were incubated with or without macrophage-skewing conditions for 24 hours, and then the upper chamber was changed to serum-free DMEM culture medium, and the lower chamber was changed to DMEM with 5\% FBS, with or without $100 \mathrm{ng} / \mathrm{ml}$ POSTN. Cells were allowed to migrate through the Transwell insert membrane for 24 hours. The inserts were removed, fixed in cold methanol, and stained with methanol containing $1 \%$ crystal violet. The inside of the Transwell was swabbed thoroughly with cotton swabs and air dried at room temperature overnight. The number of migrated raw cells and macrophages was counted by bright-field microscopy at $\times 40$. Four bright-field images were analyzed per well. Values from each well were averaged and normalized to the media control. At least 3 mice were used per condition in at least 2 separate experiments.

\section{Macrophage skewing}

Bone marrow-derived macrophages were seeded at 250,000 cells/ well in 24-well plates in DMEM medium containing 10\% FBS and 1\% penicillin-streptomycin. LPS $(100 \mathrm{ng} / \mathrm{ml})$ and IFN- $\gamma(25 \mathrm{ng} / \mathrm{ml})$ were added to the media for M1 differentiation, and IL-4 $(25 \mathrm{ng} / \mathrm{ml})$ was added to the media for M2 differentiation. After 24 hours in skewing conditions, $100 \mathrm{ng} / \mathrm{ml}$ POSTN was added to some of the cultures, and the macrophages were cultured for another 24 hours. Total RNA was extracted from the macrophages and used in qRT-PCR analyses. Cxcl9, Cxcl10, Cd86, iNos, Il12, and Tnfa were used as M1 markers, and Il1O, Ym1, Arg1, Fizz, and Mrc were used as M2 markers.

\section{HSC culture and stimulation}

Immortalized HSCs were cultured as described previously (84). HSCs were grown to $80 \%$ confluence and seeded into 12 -well plates at a density of 250,000 cells in $2 \mathrm{ml}$ growth media per well. After 24 hours at $37^{\circ} \mathrm{C}$ in a humidified $5 \% \mathrm{CO}_{2}$ incubator, serum-free media were used and cells were treated with $1 \mathrm{ng} / \mathrm{ml}$ TGF- $\beta$ (STEMCELL Technologies), $10 \mathrm{ng} / \mathrm{ml} \mathrm{NRG1}$ (Thermo Fisher Scientific), or both for 24 hours at $37^{\circ} \mathrm{C}$ with $5 \% \mathrm{CO}_{2}$. Postn expression was measured by qRT-PCR.

\section{Macrophage depletion}

AT model. Mice were treated with $200 \mu$ l clodronate or vehicle control liposomes 5 weeks after AT. Each following week, mice received $100 \mu \mathrm{l}$ clodronate or control liposomes until neuropathy developed. For the Aire ${ }^{G W /+}$ spontaneous model, mice were treated weekly for 7 weeks with $200 \mu \mathrm{l}$ clodronate or vehicle control liposomes starting at 7 weeks of age. Treatment was discontinued after 7 weeks of treatment because of the development of ascites. Clodronate and control liposomes were purchased from Encapsula NanoSciences.

\section{X-gal staining of sciatic nerves and EM}

Mice were euthanized and perfused with $0.1 \mathrm{M}$ phosphate buffer (PB) followed by cold $0.5 \%$ glutaraldehyde (Sigma-Aldrich) in $0.1 \mathrm{M} \mathrm{PB}$. Sciatic nerves were fixed in $0.5 \%$ glutaraldehyde in $0.1 \mathrm{M} \mathrm{PB}$ for 3 hours at $4^{\circ} \mathrm{C}$. Nerves were washed twice with $0.1 \mathrm{M} \mathrm{PB}$ and then twice with 2 $\mathrm{MgCl}_{2}$ in $0.1 \mathrm{M} \mathrm{PB}$. Samples were incubated overnight at $37^{\circ} \mathrm{C}$ in the following X-gal working solution: $2.0 \mathrm{mM} \mathrm{MgCl}_{2}$ (Sigma-Aldrich), $4.0 \mathrm{mM} \mathrm{K}{ }_{4} \mathrm{Fe}(\mathrm{CN})_{6}$ (Sigma-Aldrich), $4.0 \mathrm{mM} \mathrm{K} \mathrm{Ke}_{3}(\mathrm{CN})_{6}$ (SigmaAldrich), and $1.0 \mathrm{mg} / \mathrm{ml} \mathrm{X}$-gal (5-bromo-4-chloro-3-indolyl- $\beta$-Dgalactopyranoside) (Sigma-Aldrich) in 0.1 M PB. Nerves were washed twice in $0 . \mathrm{M} \mathrm{PB}$, post-fixed in $4 \%$ glutaraldehyde (Sigma-Aldrich) overnight at $4^{\circ} \mathrm{C}$, rinsed in $0.1 \mathrm{M} \mathrm{PB}$, transferred to a $2 \% \mathrm{OsO} 4$ in $0.1 \mathrm{M} \mathrm{PB}$ for 1 hour, and then processed for embedment in Epon. Semithin sections were stained with $1 \%$ paraphenylenediamine or alkaline toluidine blue and visualized by light microscopy (Leica DMR) using interactive software (Leica Application Suite). Thin sections were stained with lead citrate and imaged in a Jeol-1010 transmission electron microscope. Selected images were processed with Adobe Photoshop to generate the figures.

\section{Quantitation of demyelination}

Semithin sections of tibial nerve stained with $1 \%$ paraphenylenediamine were analyzed using Fiji software (ImageJ, NIH). The area of each tibial nerve was determined on the basis of the inner edge of the perineurium. The total number of myelinated axons in each cross section was counted manually using Fiji software to mark each myelinated axon. The following formula was used to determine the number of 
myelinated axons per 1,000 $\mu \mathrm{m}^{2}$ : (number of total axons in the tibial nerve/total area of the tibial nerve) $\times 1,000$.

\section{qRT-PCR}

Primers. The following primers were used: Arg (Mm00475988 m1), Cd86 (Mm00444543_m1), Fizz (Mm00445109_m1), iNos (Mm00440502_m1),Mrc(Mm01329362_m1),_Lama2(Mm00550083 m1), Lama4 (Mm01193660_m1), Lama5 (Mm01222029_m1), Lamb1 (Mm00801853_m1), Postn (Mm01284919_m1), POSTN (Hs01566750_m1), Thbs2 (Mm01279240_m1), Tnfa (Mm00443258_ $\mathrm{m1}$ ), and Ym1 (Mm00657889_mH). The primer probe set for cyclophilin A (82) or GAPDH (Hs02758991_g1) was used as an internal control.

Whole sciatic nerves were homogenized by mortar and pestle followed by use of the QIAshredder (QIAGEN). RNA was isolated from whole nerves using the RNeasy Plus Mini Kit or Zymo RNA MicroPrep Kit (QIAGEN). The Agilent Absolutely RNA Nanoprep Kit was used to isolate RNA for samples with fewer than $10^{4}$ cells. QIAGEN's RNeasy Plus Micro Kit was used for samples with between $10^{4}$ and $5 \times 10^{5}$ cells. SuperScript II Reverse Transcriptase (Invitrogen, Thermo Fisher Scientific) was used for cDNA synthesis. TaqMan Universal PCR Master Mix (Applied Biosystems) was used for PCR. The Applied Biosystems QuantStudio 6 Flex Real Time PCR System and QuantStudio 6 and 7 software were used for data acquisition and analysis, respectively.

\section{Western blot analysis}

Whole sciatic nerves were homogenized in Laemmli buffer. Proteins were separated by SDS-PAGE and Novex NUPAGE 4\%-12\% Bis-Tris Gel (Life Technologies, Thermo Fisher Scientific) and transferred onto methanolactivated PVDF membranes (Thermo Fisher Scientific). The blot was blocked with $5 \%$ nonfat dry milk in TBS for 1 hour at room temperature and subsequently incubated overnight at $4^{\circ} \mathrm{C}$ with polyclonal anti-POSTN (Abcam) diluted to 1:5,000 in TBS with 0.01\% TBS Tween-20 (TBST) and $5 \%$ nonfat dry milk. Blots were washed, incubated with 1:10,000 HRP-conjugated goat anti-rabbit antibody (Abcam) in blocking buffer for 1 hour at room temperature, and washed again. Blots were developed by SuperSignal West Femto Maximum Sensitivity Substrate and exposed to x-ray film. GAPDH was used as a loading control. After 3 washes for 10 minutes with TBST and 1 wash for 5 minutes in TBS, the blot was incubated with HRP-conjugated mouse anti GAPDH antibody (Thermo scientific) diluted to 1:1,000 for 1 hour at room temperature. The blot was washed and developed by SuperSignal West Femto Maximum Sensitivity Substrate and exposed to $\mathrm{x}$-ray film or imaged with a Bio-Rad ChemiDoc.

\section{Statistics}

Statistics were performed with GraphPad Prism software or R. For neuropathy incidence curves, a log-rank (Mantel-Cox) test was used. An unpaired, 2-tailed $t$ test was used when comparing 2 groups with continuous values. Welch's correction was applied when the standard deviation was different for each group. For discrete data, Fisher's exact test was performed in R. ANOVA with multiple comparisons was used when comparing 3 or more groups. A paired $t$ test was used when experimental and control samples were obtained from the same animal, as in the nerve transection surgery. For the macrophage chemotaxis assay, statistics were performed in R using the $\operatorname{lm}()$ function, in which parameters were fit to each mouse as well as the difference in treatment. $P$ values were calculated by comparing the fit of the full model to a model without a term for the difference in treatment. $P$ values were adjusted using Bonferroni's correction. A $P$ value of less than 0.05 was considered significant. Unless otherwise noted, data are presented as individual values with means.

\section{Study approval}

All animal experiments were approved by the IACUC of UNC-CH and performed in compliance with the Animal Welfare Act and NIH guidelines for the ethical care and use of animals in research.

\section{Author contributions}

DEA and MAS designed the study. DEA, YW, CJS, JFH, AH, SSS, and MAS analyzed the data. DEA, YW, JJL, BC, EWX, DS, CK, RN, CJS, EK, XLZ, and JFH performed experiments. DEA, YW, and JS performed the statistical analyses. DEA, SSS, and MAS wrote the manuscript.

\section{Acknowledgments}

Research reported in this publication was supported by the National Institute of Neurological Disorders and Stroke (RO1NS079683, MAS, principal investigator, and R01NS107851, MAS and SSS, principal investigators); the GBS/CIDP Foundation (MAS, principal investigator); a National Institute of Allergy and Infectious Diseases (NIAID) Ruth L. Kirschstein National Research Service Award (NRSA) Institutional Research Training Grant (T32 AI 007273; J.P. Ting, principal investigator); and a National Institute of Diabetes and Digestive and Kidney Diseases (NIDDK) Ruth L. Kirschstein NRSA Institutional Research Training Grant (T32 DK 007750; R.J. Falk, principal investigator). All flow cytometric experiments were carried out with the support of the UNC Flow Cytometry Core Facility. The UNC Flow Cytometry Core Facility is supported in part by a Cancer Center Core Support Grant (P30 CA016086, to the UNC Lineberger Comprehensive Cancer Center); in part by a North Carolina Biotech Center Institutional Support Grant (2012-IDG-1006); and by the Office of the Director of UNC-CH. Confocal and widefield microscopy for murine samples was performed at the UNC Neuroscience Center Microscopy Core Facility, which is supported in part by funding from the National Institute of Neurological Disorders and Stroke (NINDS) (P30 NSO45892) and a National Institute of Child Health and Human Development Intellectual and Developmental Disabilities Research Center Support Grant (U54 HD079124). The content is solely the responsibility of the authors and does not necessarily represent the official views of the NIH.

Address correspondence to: Maureen Su, Department of Microbiology Immunology and Medical Genetics (MIMG) and Pediatrics, UCLA, 615 Charles E. Young Drive South, Los Angeles, California 90095, USA. Phone: 310.825.2130; Email: masu@mednet.ucla.edu.
1. Dalakas MC, Medscape. Advances in the diagnosis, pathogenesis and treatment of CIDP. Nat Rev Neurol. 2011;7(9):507-517.

2. Goodfellow JA, Willison HJ. Guillain-Barré syndrome: a century of progress. Nat Rev Neurol. 2016;12(12):723-731.

3. Ropper AH. Current treatments for CIDP. Neurology. 2003;60(8 Suppl 3):S16-S22.
4. Hughes RA, Brassington R, Gunn AA, van Doorn PA. Corticosteroids for Guillain-Barré syndrome. Cochrane Database Syst Rev. 2016;10:CD001446.

5. Kuwabara S, Misawa S, Mori M, Tamura N, 
Kubota M, Hattori T. Long term prognosis of chronic inflammatory demyelinating polyneuropathy: a five year follow up of 38 cases. $\mathrm{J} \mathrm{Neu-}$ rol Neurosurg Psychiatry. 2006;77(1):66-70.

6. Salomon B, et al. Development of spontaneous autoimmune peripheral polyneuropathy in B7-2-deficient NOD mice. J Exp Med. 2001;194(5):677-684.

7. Su MA, et al. Defective autoimmune regulatordependent central tolerance to myelin protein zero is linked to autoimmune peripheral neuropathy. J Immunol. 2012;188(10):4906-4912.

8. Shin T, Ahn M, Matsumoto Y, Moon C. Mechanism of experimental autoimmune neuritis in Lewis rats: the dual role of macrophages. Histol Histopathol. 2013;28(6):679-684.

9. Xia RH, Yosef N, Ubogu EE. Selective expression and cellular localization of pro-inflammatory chemokine ligand/receptor pairs in the sciatic nerves of a severe murine experimental autoimmune neuritis model of Guillain-Barré syndrome. Neuropathol Appl Neurobiol. 2010;36(5):388-398.

10. Mathey EK, et al. Chronic inflammatory demyelinating polyradiculoneuropathy: from pathology to phenotype. J Neurol Neurosurg Psychiatry. 2015;86(9):973-985.

11. Brechenmacher C, et al. Guillain-Barré syndrome: an ultrastructural study of peripheral nerve in 65 patients. Clin Neuropathol. 1987;6(1):19-24.

12. Kim HJ, Jung CG, Jensen MA, Dukala D, Soliven B. Targeting of myelin protein zero in a spontaneous autoimmune polyneuropathy. JImmunol. 2008;181(12):8753-8760.

13. Hahn AF. Experimental allergic neuritis (EAN) as a model for the immune-mediated demyelinating neuropathies. Rev Neurol (Paris). 1996;152(5):328-332.

14. Li S, et al. Circulating Th17, Th22, and Th1 cells are elevated in the Guillain-Barré syndrome and downregulated by IVIg treatments. Mediators Inflamm. 2014;2014:740947.

15. Chi LJ, Xu WH, Zhang ZW, Huang HT, Zhang LM, Zhou J. Distribution of Th17 cells and Th1 cells in peripheral blood and cerebrospinal fluid in chronic inflammatory demyelinating polyradiculoneuropathy. JPeripher Nerv Syst. 2010;15(4):345-356.

16. Wu X, Wang J, Liu K, Zhu J, Zhang HL. Are Th17 cells and their cytokines a therapeutic target in Guillain-Barré syndrome? Expert Opin Ther Targets. 2016;20(2):209-222.

17. Kiefer R, Kieseier BC, Stoll G, Hartung HP. The role of macrophages in immune-mediated damage to the peripheral nervous system. Prog Neurobiol. 2001;64(2):109-127.

18. Chernousov MA, Yu WM, Chen ZL, Carey DJ, Strickland S. Regulation of Schwann cell function by the extracellular matrix. Glia. 2008;56(14):1498-1507.

19. Barros CS, Franco SJ, Müller U. Extracellular matrix: functions in the nervous system. Cold Spring Harb Perspect Biol. 2011;3(1):a005108.

20. Uchida M, et al. Periostin, a matricellular protein, plays a role in the induction of chemokines in pulmonary fibrosis. Am J Respir Cell Mol Biol. 2012;46(5):677-686

21. Masuoka M, et al. Periostin promotes chronic allergic inflammation in response to Th2 cyto- kines. J Clin Invest. 2012;122(7):2590-2600.

22. Prakoura N, Kavvadas P, Kormann R, Dussaule JC, Chadjichristos CE, Chatziantoniou C. $\mathrm{NF \kappa B}$-induced periostin activates integrin- $\beta 3$ signaling to promote renal injury in GN. J Am Soc Nephrol. 2017;28(5):1475-1490.

23. Yokota K, et al. Periostin promotes scar formation through the interaction between pericytes and infiltrating monocytes/macrophages after spinal cord injury. Am J Pathol. 2017;187(3):639-653.

24. Gaudet AD, Popovich PG. Extracellular matrix regulation of inflammation in the healthy and injured spinal cord. Exp Neurol. 2014;258:24-34.

25. Schmidt B, Toyka KV, Kiefer R, Full J, Hartung HP, Pollard J. Inflammatory infiltrates in sural nerve biopsies in Guillain-Barre syndrome and chronic inflammatory demyelinating neuropathy. Muscle Nerve. 1996;19(4):474-487.

26. Cornblath DR, Griffin DE, Welch D, Griffin JW, McArthur JC. Quantitative analysis of endoneurial T-cells in human sural nerve biopsies. J Neuroimmunol. 1990;26(2):113-118.

27. Bouchard C, et al. Clinicopathologic findings and prognosis of chronic inflammatory demyelinating polyneuropathy. Neurology. 1999;52(3):498-503.

28. Csurhes PA, Sullivan AA, Green K, Pender MP, McCombe PA. T cell reactivity to PO, P2, PMP-22, and myelin basic protein in patients with Guillain-Barre syndrome and chronic inflammatory demyelinating polyradiculoneuropathy. J Neurol Neurosurg Psychiatry. 2005;76(10):1431-1439.

29. Madia F, et al. pSTAT1, pSTAT3, and T-bet as markers of disease activity in chronic inflammatory demyelinating polyradiculoneuropathy. J Peripher Nerv Syst. 2009;14(2):107-117.

30. Dalakas MC. Pathogenesis of immunemediated neuropathies. Biochim Biophys Acta. 2015;1852(4):658-666.

31. Yang D, et al. Coordinate control of axon defasciculation and myelination by laminin-2 and -8 . J Cell Biol. 2005;168(4):655-666.

32. Occhi S, et al. Both laminin and Schwann cell dystroglycan are necessary for proper clustering of sodium channels at nodes of Ranvier. J Neurosci. 2005;25(41):9418-9427.

33. Adams JC, Tucker RP. The thrombospondin type 1 repeat (TSR) superfamily: diverse proteins with related roles in neuronal development. Dev Dyn. 2000;218(2):280-299.

34. Lindsley A, et al. Identification and characterization of a novel Schwann and outflow tract endocardial cushion lineage-restricted periostin enhancer. Dev Biol. 2007;307(2):340-355.

35. Sonnenberg-Riethmacher E, Miehe M, Riethmacher D. Promotion of periostin expression contributes to the migration of Schwann cells. J Cell Sci. 2015;128(17):3345-3355.

36. Hoffman JR, O'Shea KS. Thrombospondin expression in nerve regeneration I. Comparison of sciatic nerve crush, transection, and long-term denervation. Brain Res Bull. 1999;48(4):413-420.

37. Sorocos K, Kostoulias X, Cullen-McEwen L, Hart $\mathrm{AH}$, Bertram JF, Caruana G. Expression patterns and roles of periostin during kidney and ureter development. J Urol. 2011;186(4):1537-1544.

38. Norris RA, et al. Periostin regulates collagen fibrillogenesis and the biomechanical properties of connective tissues. J Cell Biochem.
2007;101(3):695-711.

39. Suzuki H, et al. Immunohistochemical localization of periostin in tooth and its surrounding tissues in mouse mandibles during development. Anat Rec A Discov Mol Cell Evol Biol. 2004;281(2):1264-1275.

40. Norris RA, Kern CB, Wessels A, Moralez EI, Markwald RR, Mjaatvedt $\mathrm{CH}$. Identification and detection of the periostin gene in cardiac development. Anat Rec A Discov Mol Cell Evol Biol. 2004;281(2):1227-1233.

41. Johansson MW, Annis DS, Mosher DF. $\alpha(\mathrm{M}) \beta(2)$ integrin-mediated adhesion and motility of IL-5stimulated eosinophils on periostin. Am J Respir Cell Mol Biol. 2013;48(4):503-510.

42. Gillan L, Matei D, Fishman DA, Gerbin CS, Karlan BY, Chang DD. Periostin secreted by epithelial ovarian carcinoma is a ligand for alpha(V)beta(3) and alpha(V)beta(5) integrins and promotes cell motility. Cancer Res. 2002;62(18):5358-5364.

43. Koh SJ, et al. Matricellular Protein Periostin Mediates Intestinal Inflammation through the Activation of Nuclear Factor $\kappa \mathrm{B}$ Signaling. PLoS One. 2016;11(2):e0149652.

44. Louvet C, et al. A novel myelin PO-specific T cell receptor transgenic mouse develops a fulminant autoimmune peripheral neuropathy. J Exp Med. 2009;206(3):507-514.

45. Oka T, et al. Genetic manipulation of periostin expression reveals a role in cardiac hypertrophy and ventricular remodeling. Circ Res. 2007;101(3):313-321.

46. Feltri ML, Scherer SS, Wrabetz L, Kamholz J, Shy ME. Mitogen-expanded Schwann cells retain the capacity to myelinate regenerating axons after transplantation into rat sciatic nerve. Proc Natl Acad Sci U S A. 1992;89(18):8827-8831.

47. Jessen KR, Morgan L, Stewart HJ, Mirsky R. Three markers of adult non-myelin-forming Schwann cells, 217c(Ran-1), A5E3 and GFAP: development and regulation by neuron-Schwann cell interactions. Development. 1990;109(1):91-103.

48. Taniuchi M, Clark HB, Schweitzer JB, Johnson EM. Expression of nerve growth factor receptors by Schwann cells of axotomized peripheral nerves: ultrastructural location, suppression by axonal contact, and binding properties. JNeurosci. 1988;8(2):664-681.

49. Lemke G, Chao M. Axons regulate Schwann cell expression of the major myelin and NGF receptor genes. Development. 1988;102(3):499-504.

50. Toews AD, et al. Primary demyelination induced by exposure to tellurium alters Schwann cell gene expression: a model for intracellular targeting of NGF receptor. J Neurosci.1992;12(9):3676-3687.

51. Meyer zu Hörste G, et al. Mouse Schwann cells activate MHC class I and II restricted T-cell responses, but require external peptide processing for MHC class II presentation. Neurobiol Dis. 2010;37(2):483-490.

52. Meyer Zu Horste G, et al. Expression of antigen processing and presenting molecules by Schwann cells in inflammatory neuropathies. Glia. 2010;58(1):80-92.

53. Hartlehnert M, et al. Schwann cells promote post-traumatic nerve inflammation and neuropathic pain through MHC class II. Sci Rep. 
2017;7(1):12518.

54. Zhou W, et al. Periostin secreted by glioblastoma stem cells recruits M2 tumour-associated macrophages and promotes malignant growth. Nat Cell Biol. 2015;17(2):170-182.

55. Katzav A, Bina H, Aronovich R, Chapman J. Treatment for experimental autoimmune neuritis with clodronate (Bonefos). Immunol Res. 2013;56(2-3):334-340.

56. Jun HS, Yoon CS, Zbytnuik L, van Rooijen N, Yoon JW. The role of macrophages in T cell-mediated autoimmune diabetes in nonobese diabetic mice. JExp Med.1999;189(2):347-358.

57. Tran EH, Hoekstra K, van Rooijen N, Dijkstra $\mathrm{CD}$, Owens T. Immune invasion of the central nervous system parenchyma and experimental allergic encephalomyelitis, but not leukocyte extravasation from blood, are prevented in macrophage-depleted mice. JImmunol. 1998;161(7):3767-3775.

58. Zhou D, et al. Critical involvement of macrophage infiltration in the development of Sjögren's syndrome-associated dry eye. Am J Pathol. 2012;181(3):753-760.

59. Izuhara K, et al. Periostin in inflammation and allergy. Cell Mol Life Sci. 2017;74(23):4293-4303.

60. Cui D, Huang Z, Liu Y, Ouyang G. The multifaceted role of periostin in priming the tumor microenvironments for tumor progression. Cell Mol Life Sci. 2017;74(23):4287-4291.

61. Hakuno D, et al. Periostin advances atherosclerotic and rheumatic cardiac valve degeneration by inducing angiogenesis and MMP production in humans and rodents. JClin Invest. 2010;120(7):2292-2306.

62. O'Dwyer DN, Moore BB. The role of periostin in lung fibrosis and airway remodeling. Cell Mol Life Sci. 2017;74(23):4305-4314.

63. Horiuchi $\mathrm{K}$, et al. Identification and characterization of a novel protein, periostin, with restricted expression to periosteum and periodontal ligament and increased expression by transforming growth factor beta. J Bone Miner Res. 1999;14(7):1239-1249.

64. Amara S, et al. Synergistic effect of pro-inflammatory TNF $\alpha$ and IL-17 in periostin mediated collagen deposition: potential role in liver fibrosis. Mol Immunol. 2015;64(1):26-35.

65. Shih CH, Lacagnina M, Leuer-Bisciotti K, Pröschel C. Astroglial-derived periostin promotes axonal regeneration after spinal cord injury. J Neurosci. 2014;34(7):2438-2443.

66. Irie S, Kashiwabara M, Yamada A, Asano K. Suppressive Activity of Quercetin on Periostin Functions In Vitro. In Vivo. 2016;30(1):17-25.

67. Perry VH, Brown MC, Gordon S. The macrophage response to central and peripheral nerve injury. A possible role for macrophages in regeneration. JExp Med.1987;165(4):1218-1223.

68. Perry VH, Brown MC. Role of macrophages in peripheral nerve degeneration and repair. Bioessays. 1992;14(6):401-406.

69. Xue J, et al. Transcriptome-based network analysis reveals a spectrum model of human macrophage activation. Immunity. 2014;40(2):274-288

70. Martini R, Toyka KV. Immune-mediated components of hereditary demyelinating neuropathies: lessons from animal models and patients. Lancet Neurol. 2004;3(8):457-465.

71. DeFrancesco-Lisowitz A, Lindborg JA, Niemi JP, Zigmond RE. The neuroimmunology of degeneration and regeneration in the peripheral nervous system. Neuroscience. 2015;302:174-203.

72. Perrin FE, Lacroix S, Avilés-Trigueros M, David S. Involvement of monocyte chemoattractant protein-1, macrophage inflammatory protein1alpha and interleukin-1beta in Wallerian degeneration. Brain. 2005;128(Pt 4):854-866.

73. Schreiber RC, et al. Monocyte chemoattractant protein (MCP)-1 is rapidly expressed by sympathetic ganglion neurons following axonal injury. Neuroreport. 2001;12(3):601-606.

74. Tanaka T, Minami M, Nakagawa T, Satoh M.
Enhanced production of monocyte chemoattractant protein-1 in the dorsal root ganglia in a rat model of neuropathic pain: possible involvement in the development of neuropathic pain. Neurosci Res. 2004;48(4):463-469.

75. Liu L, et al. Role of Periostin in Early Brain Injury After Subarachnoid Hemorrhage in Mice. Stroke. 2017;48(4):1108-1111.

76. Liu GX, Xi HQ, Sun XY, Wei B. Role of periostin and its antagonist PNDA-3 in gastric cancer metastasis. World J Gastroenterol. 2015;21(9):2605-2613.

77. Zhu M, et al. Neutralizing monoclonal antibody to periostin inhibits ovarian tumor growth and metastasis. Mol Cancer Ther. 2011;10(8):1500-1508.

78. O'Day SJ, et al. Clinical and pharmacologic evaluation of two dose levels of intetumumab (CNTO 95) in patients with melanoma or angiosarcoma. Invest New Drugs. 2012;30(3):1074-1081.

79. Nemeth JA, et al. Alpha-v integrins as therapeutic targets in oncology. Cancer Invest. 2007;25(7):632-646.

80. Su MA, et al. Mechanisms of an autoimmunity syndrome in mice caused by a dominant mutation in Aire. JClin Invest. 2008;118(5):1712-1726.

81. Xia RH, Yosef N, Ubogu EE. Dorsal caudal tail and sciatic motor nerve conduction studies in adult mice: technical aspects and normative data. Muscle Nerve. 2010;41(6):850-856.

82. Zeng XL, Nagavalli A, Smith CJ, Howard JF, Su MA. Divergent effects of T cell costimulation and inflammatory cytokine production on autoimmune peripheral neuropathy provoked by Aire deficiency. J Immunol. 2013;190(8):3895-3904.

83. Sensken SC, Nagarajan M, Bode C, Gräler MH. Local inactivation of sphingosine 1-phosphate in lymph nodes induces lymphopenia. JImmunol. 2011;186(6):3432-3440.

84. Lehmann HC, et al. Human Schwann cells retain essential phenotype characteristics after immortalization. Stem Cells Dev. 2012;21(3):423-431. 